\title{
Spatially bivariate EEG-neurofeedback can manipulate interhemispheric rebalancing of M1 excitability
}

\section{Authors:}

Masaaki Hayashi ${ }^{1}$, Kohei Okuyama ${ }^{2}$, Nobuaki Mizuguchi ${ }^{3}$, Ryotaro Hirose ${ }^{1}$, Taisuke Okamoto $^{1}$, Michiyuki Kawakami ${ }^{2}$, Junichi Ushiba ${ }^{4 *}$

\section{Affiliations:}

$9{ }^{1}$ Graduate School of Science and Technology, Keio University, Kanagawa, Japan; ${ }^{2}$ Department of Rehabilitation Medicine, School of Medicine, Keio University, Tokyo, Japan; ${ }^{3}$ Research Organization of Science and Technology, Ritsumeikan University, 2 Shiga, Japan; ${ }^{4}$ Faculty of Science and Technology, Keio University, Kanagawa, Japan.

4 *Correspondence:

5 Junichi Ushiba, $\mathrm{PhD}$

6 Department of Biosciences and Informatics, Faculty of Science and Technology, Keio

17 University, 3-14-1 Hiyoshi, Kouhoku-ku, Yokohama, Kanagawa 223-8522, Japan.

8 Tel/Fax: +81-45-566-1678.

9 Email: ushiba@bio.keio.ac.jp

1 Junichi Ushiba is the Founder and Representative Director of the University Startup

2 Company Connect Inc. involved in the research, development, and sales of

23 rehabilitation devices, including brain-computer interfaces. He receives a salary from

4 Connect Inc. and holds shares in Connect Inc. This company does not have any

5 relationships with the device or setup used in the current study. M. Hayashi is employed 6 by the company. 


\section{Abstract}

30 Human behavior requires interregional crosstalk to employ the sensorimotor processes in 31 the brain. Although some external neuromodulation tools have been used to manipulate 32 interhemispheric sensorimotor activity, a central controversy concerns whether this activity can be volitionally controlled. Experimental tools lack the power to up- or downregulate the state of the targeted hemisphere over a large dynamic range and, therefore, cannot evaluate the possible volitional control of the activity. We overcame this difficulty by using the recently developed method of spatially bivariate electroencephalography (EEG)-neurofeedback to systematically enable participants to manipulate their bilateral sensorimotor activities. Herein, we report that bi-directional changes in ipsilateral excitability to the imagined hand (conditioning hemisphere) affect interhemispheric inhibition (IHI) assessed by paired-pulse transcranial magnetic stimulation paradigm. In addition, participants were able to robustly manipulate the IHI magnitudes. Further physiological analyses revealed that the self-manipulation of IHI magnitude reflected interhemispheric connectivity in EEG and TMS, which was accompanied by intrinsic bilateral cortical oscillatory activities. Our results provide clear neuroscientific evidence regarding the inhibitory interhemispheric sensorimotor activity and IHI manipulator, thereby challenging the current theoretical concept of recovery of motor function for neurorehabilitation.

Keywords: Interhemispheric inhibition, brain-computer interface, neurofeedback, neural manipulation, inhibitory sensorimotor processes

\section{Abbreviations}

53 Electroencephalogram, EEG; transcranial magnetic stimulation, TMS; interhemispheric inhibition, IHI; brain-computer interface, BCI; sensorimotor rhythms, SMR; eventrelated desynchronization/synchronization, (ERD/ERS); sensorimotor cortex, SM1; motor-evoked potential, MEP; motor imagery, MI; first dorsal interosseous, FDI; abductor digiti minimi, ADM; standard deviation, SD; repeated-measures ANOVA, rmANOVA; laterality index, LI; corrected imaginary part of coherence, $\mathrm{ciCOH}$; functional magnetic resonance imaging, fMRI; corticospinal tract, CST; electromyogram, 


\section{Introduction}

Projection neurons wire the brain over long distances and provide a network between different brain regions. In particular, both hemispheres are structurally connected by transcallosal projections and exhibit functional cross talks (Hofer and Frahm, 2006; Meyer et al., 1995); such interhemispheric interaction is essential for higher order cognitive and sensorimotor brain functions. An early argument for interhemispheric interaction, rather than the specific processes performed by each brain area, was that dynamic interplay via the callosum not only allows for simple coordination of processing between the hemispheres but also has profound effects on attentional functioning (Banich, 1998). In the motor domain, neurons in the monkey primary motor cortex are fired bilaterally and motor signals from the two hemispheres interact during unimanual motor tasks (Ames and Churchland, 2019); in the human motor cortex, measuring these neural activities via electroencephalography (EEG) and paired-pulse transcranial magnetic stimulation (TMS) recently revealed that the two hemispheres act together and the related cortical oscillatory activity influences the inhibitory interhemispheric brain network (Picazio et al., 2014; Stefanou et al., 2018).

A critical issue is whether a specific interhemispheric activity can be volitionally controlled. Previous studies have focused on inhibitory interhemispheric sensorimotor network, represented by interhemispheric inhibition (IHI), from movement-related manner (Duque et al., 2007, 2005; Murase et al., 2004), and passive neuromodulation effects by externally administered intervention using transcranial direct current stimulation (tDCS) or repetitive transcranial magnetic stimulation (rTMS) (Boddington and Reynolds, 2017; Gilio et al., 2003; Williams et al., 2010). Due to the experimental limitations related to these observational or open-loop paradigms, much less is known about the effects of changes in sensorimotor activity patterns in both hemispheres on IHI, and the possibility of consciously self-manipulating the inhibitory interhemispheric sensorimotor activity. Therefore, the identification of bilateral sensorimotor activity patterns that underlie IHI and the potent self-manipulator of IHI should be of great value for the understanding of human sensorimotor neural plasticity (Figure 1A).

As a recent neural manipulative tool, numerous studies have used a closed-loop brain-computer interface (BCI)-based neurofeedback whereby participants learn to volitionally desynchronize and synchronize oscillatory sensorimotor rhythms (SMRERD/ERS) in the contralateral sensorimotor cortex (SM1) through visual and/or somatosensory feedback (Ang and Guan, 2017; Chaudhary et al., 2016; Ramos- 
97 Murguialday et al., 2013; Soekadar et al., 2015b). BCI-based neurofeedback is supported 98 by the fact that the intensity of SMR-ERD in EEG represents states of high versus low excitability of not only the sensorimotor cortex (Neuper et al., 2006; Neuper and

100 Pfurtscheller, 2001; Pfurtscheller et al., 2006), but also the corticospinal descending 101 pathway, as measured by the motor-evoked potential (MEP) amplitude (Takemi et al., 102 2015, 2013). Because both hemispheres are structurally and functionally connected, it is 103 likely that the balance of bilateral SMR-ERDs and transcallosal excitability states are 104 linked, as indicated by common variation of conditioning MEP amplitude and IHI 105 (Ferbert et al., 1992; Ghosh et al., 2013; Ni et al., 2009).

106 Here, we sought to investigate the association of IHI and bilateral SMR-ERDs to 107 understand the inhibitory sensorimotor functions of interhemispheric interaction that may 108 critically depend on the oscillatory brain activity in both hemispheres. Furthermore, if the oscillatory brain activity reflected IHI, we tested whether it can be self-manipulated using 110 a dual-coil paired-pulse TMS protocol (Daskalakis et al., 2002; Ferbert et al., 1992). To 111 assess the association of IHI with ongoing oscillatory brain activity, we used a recently 112 developed spatially bivariate BCI-based neurofeedback technique that allows volitional 113 modulation of SMR amplitude in both hemispheres (Hayashi et al., 2021, 2020) and 114 triggers the TMS pulses in real time at pre-specified bilateral SMR amplitudes. Our 115 system, therefore, overcomes previously unresolved experimental limitations; unlike 116 the ordinary observational or open-loop experimental paradigm, our paradigm 117 enables participants to control the bivariate sensorimotor excitability, evaluates the 118 inhibitory sensorimotor functions that underlie IHI, and determines whether it is 119 possible to consciously self-manipulate the IHI magnitude.

120 Using the novel closed-loop bi-hemispheric brain state-dependent EEG-triggered 121 dual-coil TMS system (bi-EEG-triggered dual-TMS), we evaluated the effects of 122 different states of the targeted bidirectional up- or down-conditioned one-sided 123 hemisphere on effective inhibitory interhemispheric network expressed by IHI: (1) 124 resting state, (2) during motor imagery without visual feedback, (3) high, (4) middle, and 125 (5) low excitability states of the ipsilateral SM1 (conditioning side) to the unilateral 126 imagined hand movement during BCI-based neurofeedback (Figure 1B). We 127 hypothesized that if the ipsilateral dominant SMR-ERD is a potent up- or down-regulator 128 of IHI from the ipsilateral side, the strongest IHI will occur with high excitability state of 129 the ipsilateral SM1. This multimodal research provides strong evidence for the dynamic 130 interplay between distinct regions underlying IHI through BCI-based neurofeedback, and 
A

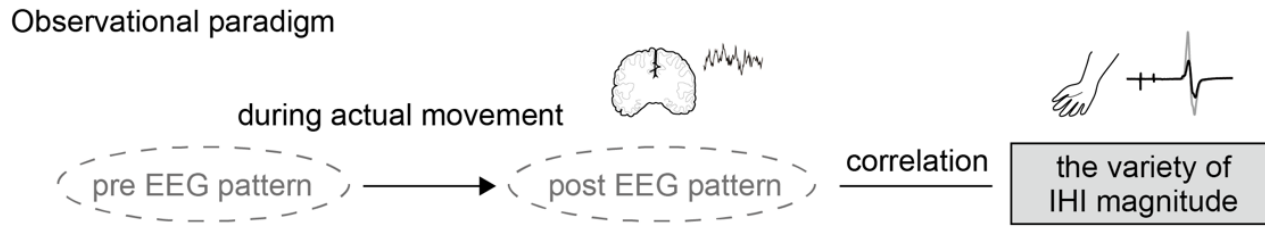

Open-loop paradigm

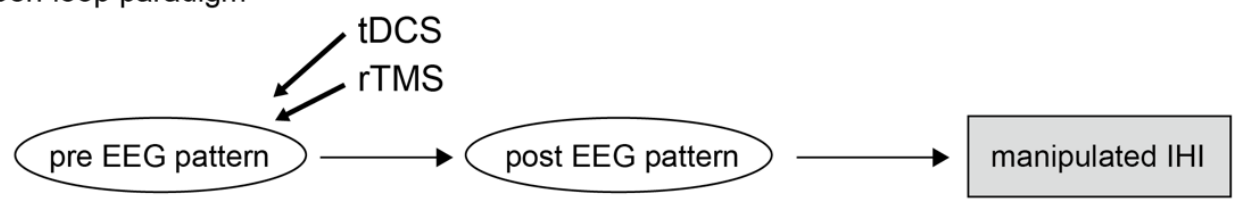

Closed-loop paradigm

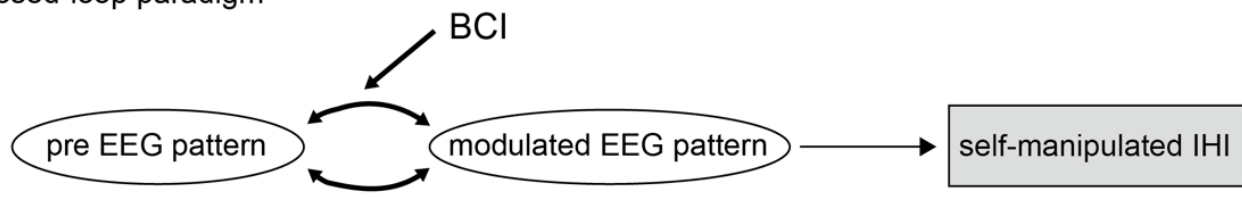

B

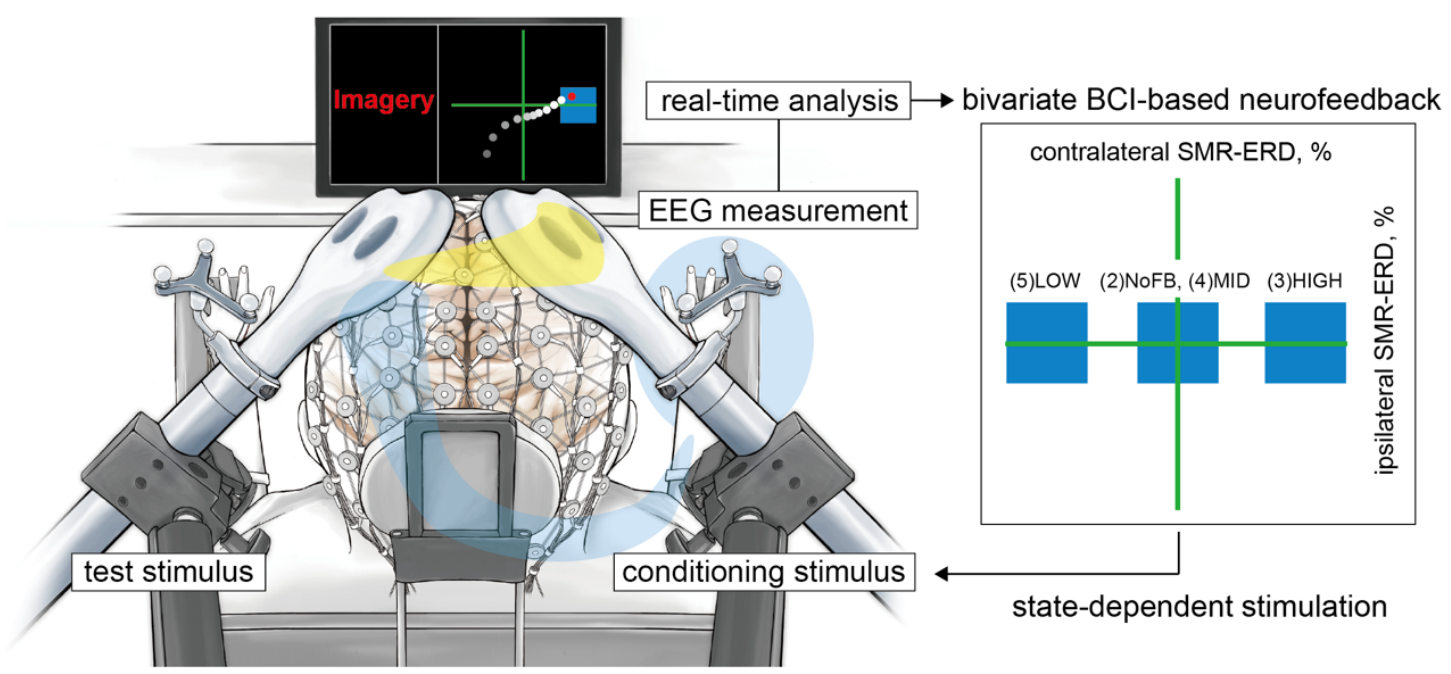

135 Figure 1 Conceptual illustration of the current study and experimental overview

136 (A) When a certain stimulus was input into the system, the brain was considered to vary with the

137 state, resulting in IHI changes. The upper panel highlights the experimental limitations in the

138 observational paradigm due to a variety of IHI magnitudes is observed during actual movement.

139 In this case, it is unclear whether the changes in EEG patterns in both hemispheres would affect

140 IHI. The middle panel indicates that it is unclear whether it is possible to consciously self-

141 manipulate inhibitory interhemispheric sensorimotor activity in the open-loop neuromodulation

142 paradigm using tDCS or rTMS. The lower panel shows that specific EEG patterns are associated

143 with IHI magnitude, and closed-loop BCI-based neurofeedback modulates the EEG activities. 
144 Therefore, if bilateral EEG patterns that underlie IHI are identified, we should be able to

145 volitionally up- and down-regulate the IHI magnitude via BCI-based neurofeedback, suggesting

146 the possibility of plastic interhemispheric balancing. (B) The current bi-EEG-triggered dual-TMS

147 experimental system involved spatially bivariate BCI-based neurofeedback that allows volitional

148 modulation of EEG patterns only in a targeted hemisphere to enable us to verify our hypothesis.

149 Different states of the targeted bidirectional up- and down-conditioned ipsilateral hemisphere to

150 the imagined hand were tested in the following states: (1) resting state, (2) during motor imagery

151 without visual feedback, (3) high, (4) middle, and (5) low excitability states. A blue target box,

152 based on the predetermined SMR-ERDs, was displayed corresponding to each session. A cue

153 signal was generated to trigger the conditioning stimulus when the signal reached the target box.

154 The yellow line on the head represents the signal flow from the conditioning hemisphere that

155 modifies the contralateral side through the corpus callosum, and the blue line represents the test

156 signal towards the right hand.

157

\section{Results}

159 The data compliance is described in the Supplementary files ("Data compliance" and "IHI 160 curves").

161

\section{IHI manipulation via spatially bivariate BCI-based neurofeedback}

163 We compared the IHI magnitude between five sessions as follows: (1) resting-state 164 (REST); (2) right finger motor imagery (MI) without visual feedback (NoFB), (3) high 165 excitability states of the ipsilateral SM1 during BCI-based neurofeedback (HIGH); (4) middle excitability states of the ipsilateral SM1 during BCI-based neurofeedback (MID); and (5) low excitability states of the ipsilateral SM1 during BCI-based neurofeedback (LOW). Typical examples of MEP amplitude elicited by single test stimulus (TS-only) and paired-pulse stimulation (conditioning stimulus [CS]+TS) of a representative participant are shown in Figure 2A. The manipulation range of IHI (i.e., the difference between HIGH and LOW sessions) was $32.6 \pm 30.7 \%$ (Cohen's $d=1.50$ ). A one-way repeated-measures ANOVA (rmANOVA) of the sessions (five levels: REST, NoFB, HIGH, MID, and LOW) revealed significant differences $\left(\mathrm{F}_{(4,88)}=6.85, p<0.001, \eta^{2}=\right.$

174 0.22; REST: $69.0 \pm 22.6 \%$, NoFB: $85.9 \pm 18.3 \%$, HIGH: $62.0 \pm 23.5 \%$, MID: $85.5 \pm$ $17526.2 \%$, LOW: $96.9 \pm 27.7 \%$ ). Across the three BCI-based neurofeedback sessions (i.e., 176 HIGH, MID, and LOW sessions) for the comparison of IHI magnitude, a post-hoc two177 tailed paired t-test showed significant difference between HIGH and MID sessions 
178 (difference $=23.5$, Cohen's $d=0.94, p=0.025$ ), and between HIGH and LOW sessions

179 (difference $=34.9$, Cohen's $d=1.36, p=0.001)$, but not between MID and LOW sessions

180 (difference $=11.4$, Cohen's $d=0.42, p=0.424$; Figure 2B). Additional results are

181 presented in Figure 2B. Importantly, rmANOVA for TS-only revealed no significant

182 differences in the MEP amplitude between all sessions $\left(\mathrm{F}_{(4,88)}=2.44, p=0.104, \eta^{2}=\right.$

183 0.09; REST: $0.98 \pm 0.45 \mathrm{mV}$, NoFB: $1.50 \pm 1.02 \mathrm{mV}$, HIGH: $1.94 \pm 1.37 \mathrm{mV}$, MID: 1.67

$184 \pm 1.15 \mathrm{mV}$, LOW: $1.93 \pm 1.41 \mathrm{mV}$ ), suggesting that participants could learn to

185 volitionally increase or decrease (bidirectional) the ipsilateral sensorimotor excitability

186 while maintaining constant contralateral sensorimotor excitability (Figure 2C).

187 In the control muscle (abductor digiti minimi, ADM), such observed modulation

188 was not driven ("IHI manipulation for control muscle" in Supplementary files). A linear

189 mixed-effect model that considered the contralateral SMR-ERD and ipsilateral SMR-

190 ERD as fixed effect, and participants as random effect revealed a significant effect of the 191 ipsilateral SMR-ERD $(p<0.001)$ on IHI magnitude, but no main effect of the 192 contralateral SMR-ERD or contralateral SMR-ERD $\times$ ipsilateral SMR-ERD interaction 193 ( $p=0.828$ and $p=0.058$, respectively). We further compared the IHI magnitude across 194 sessions by normalizing IHI magnitude to baseline (i.e., NoFB session), and calculating 195 the difference between REST, HIGH, MID, and LOW sessions. The results were 196 compatible with those presented in Figure 2 ("Comparison of normalized IHI magnitude" 197 in Supplementary files). The results of the non-triggered TMS trials are also shown in 198 the Supplementary files ("Comparison of IHI magnitude in non-triggered TMS trials") to 199 present the influence of spontaneous SMR fluctuations on IHI. 
A

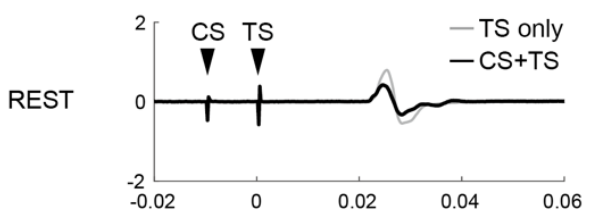

NoFB

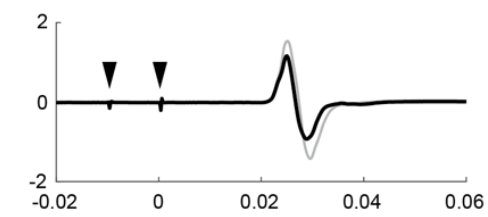

$\mathrm{HIGH}$

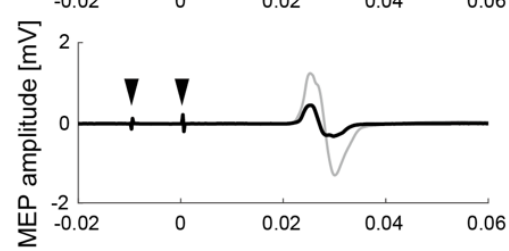

MID

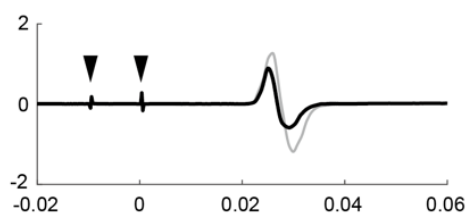

LOW

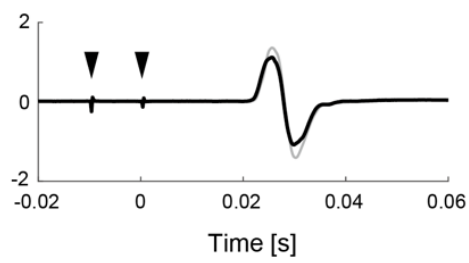

B

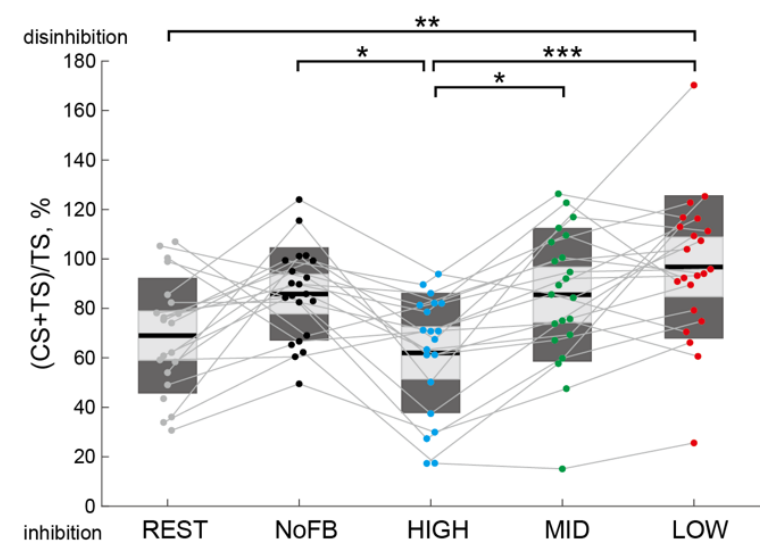

C

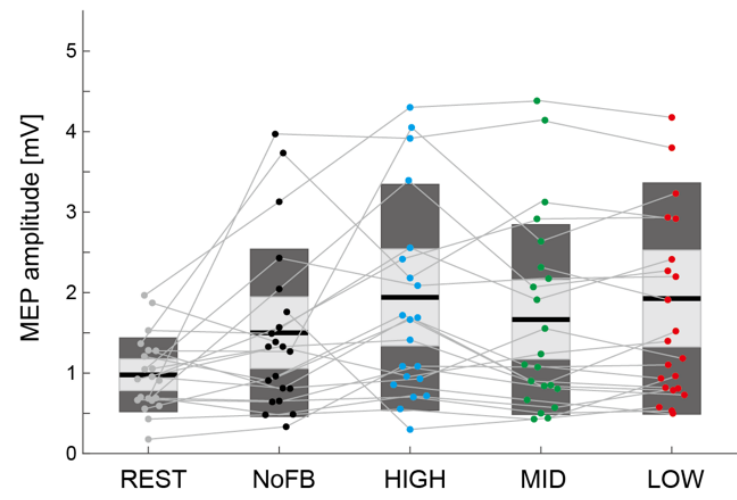

Figure 2 Comparison of IHI magnitude

(A) Typical examples of mean MEP amplitudes elicited by single TS (TS-only; light grey color) and paired-pulse stimulation (CS+TS; black color) in a representative participant. The black arrows represent the stimulus timings of CS and TS. (B) The IHI magnitude of the individual participants are represented by colored plots and thin grey lines. The figure also provides individual data in addition to the box plot. The light grey box represents 1.96 SEM (95\% confidence interval) and dark grey box represents $1 \mathrm{SD}$. The black line indicates the group mean of the studied sample and colored plots represent a single session. Lower values represent greater inhibitory effect from the ipsilateral hemisphere and therefore greater acceleration of the IHI

211 magnitude. Dendrograms above the bars represent the results of the post-hoc analyses. ${ }^{*} p<0.05$,

$212 * * p<0.01$, and $* * * p<0.001$; all comparisons were Bonferroni corrected. (C) The figure shows 213 MEP amplitude elicited by a single TS (TS-only). No significant difference in MEP amplitude 214 was observed between sessions (all $p>0.05$ ).

Modulation effects of bilateral SM1 at EEG level

217 We tested whether participants could learn volitional modulation in both hemispheres at

218 the EEG level using a spatially bivariate BCI-based neurofeedback. For the contralateral 219 SMR-ERD, a one-way rmANOVA for the sessions (five levels: REST, NoFB, HIGH, 
220 MID, and LOW) revealed a significant difference $\left(\mathrm{F}_{(4,96)}=4.81, p=0.014, \eta^{2}=0.16\right.$;

221 REST: $-10.6 \pm 11.6 \%$, NoFB: $13.5 \pm 29.5 \%$, HIGH: $19.3 \pm 26.5 \%$, MID: $16.1 \pm 24.1 \%$,

222 LOW: $13.3 \pm 28.4 \%$ ). A post-hoc two-tailed paired t-test demonstrated no significant

223 differences in the contralateral SMR-ERD between the neurofeedback sessions (HIGH-

224 MID: difference $=-3.2$, Cohen's $d=0.13, p=1.00$; HIGH-LOW: difference $=-6.0$,

225 Cohen's $d=0.22, p=1.00$; MID-LOW: difference $=-2.8$, Cohen's $d=0.11, p=1.00$,

226 whereas significant differences were found between REST and other sessions (all $p<$

227 0.05; Figure 3A). In contrast, after showing significant differences in the ipsilateral SMR-

228 ERD between sessions $\left(\mathrm{F}_{(4,96)}=156.0, p<0.001, \eta^{2}=0.86\right.$; REST: $-18.8 \pm 12.2 \%$,

229 NoFB: $-10.5 \pm 34.0 \%$, HIGH: $64.5 \pm 14.0 \%$, MID: $10.1 \pm 20.3 \%$, LOW: $-156.3 \pm$

$23049.0 \%$ ), a post-hoc two-tailed paired t-test showed that ipsilateral SMR-ERD increased

231 during the HIGH session (HIGH-MID: difference $=-54.4$, Cohen's $d=3.12, p<0.001$;

232 HIGH-LOW: difference $=-220.8$, Cohen's $d=6.13, p<0.001$ ) and decreased during the

233 LOW session (MID-LOW: difference $=-166.4$, Cohen's $d=4.44, p<0.001$ ), revealing

234 a significant modulation effect compared to baseline sensorimotor endogenous activity

235 (Figure 3B). To investigate whether self-modulated changes in bilateral SMR-ERDs were

236 specific to the hemisphere targeted by spatially bivariate BCI-based neurofeedback, we

237 analyzed the laterality index (LI) for each session. A one-way rmANOVA for the sessions

238 (five levels: REST, NoFB, HIGH, MID, and LOW) revealed significant differences

$239\left(\mathrm{~F}_{(4,96)}=53.9, p<0.001, \eta^{2}=0.68\right.$; REST: $-0.14 \pm 0.34$, NoFB: $-0.28 \pm 0.31$, HIGH:

$2400.54 \pm 0.27$, MID: $-0.09 \pm 0.42$, LOW: $-0.89 \pm 0.14)$. The post-hoc two-tailed paired t-

241 tests demonstrated that LI increased during the HIGH session (HIGH-MID: difference =

$242-0.63$, Cohen's $d=1.78, p<0.001$; HIGH-LOW: difference $=-1.43$, Cohen's $d=6.65, p$

$243<0.001$ ) and decreased during the LOW session (MID-LOW: difference $=-0.80$, Cohen's

$244 d=2.56, p<0.001$ ), indicating that the balance of interhemispheric sensorimotor

245 excitability was modulated (Figure 3C). The spatial patterns of SMR-ERD in each session

246 are depicted in Figure 3D. We found that the SMR-ERDs were predominantly localized

247 in bilateral parieto-temporal regions (around the $\mathrm{C} 3$ and $\mathrm{C} 4$ channels and their periphery),

248 and strong ipsilateral SMR-ERD was observed in the HIGH session with constant 249 contralateral SMR-ERD. 

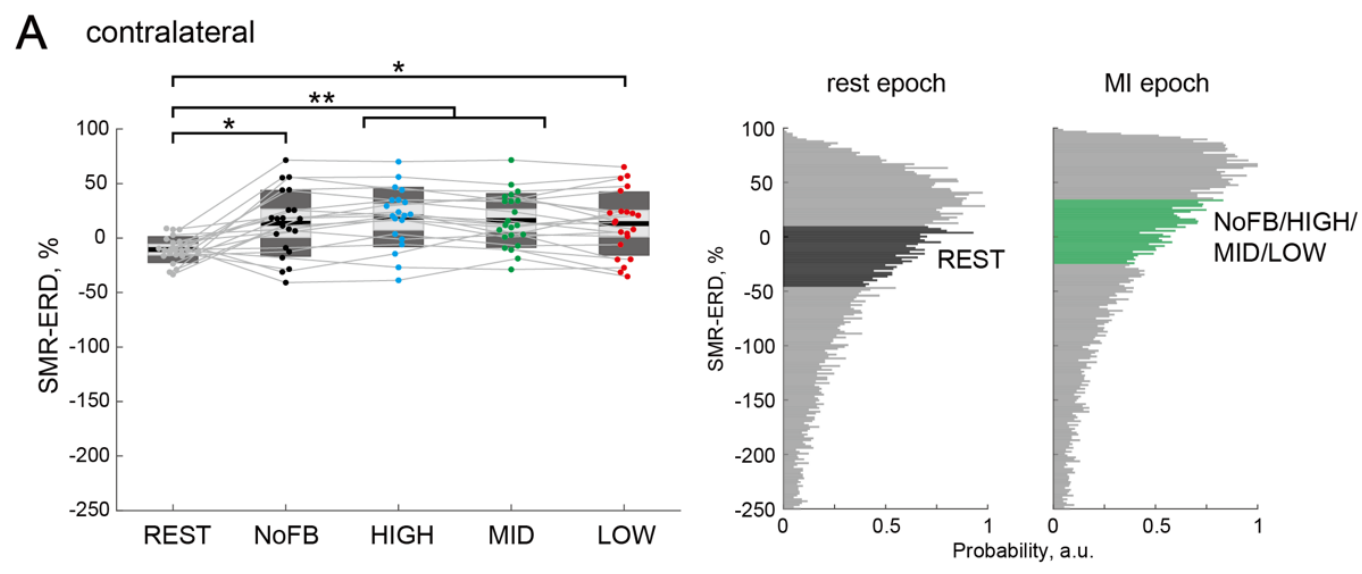

B ipsilateral
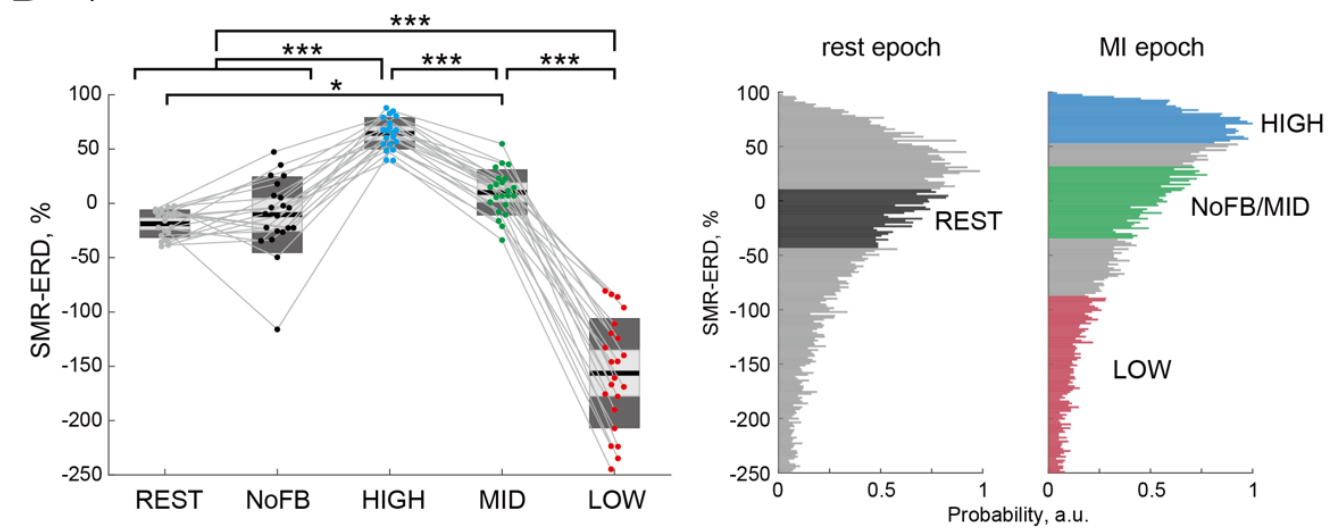

C
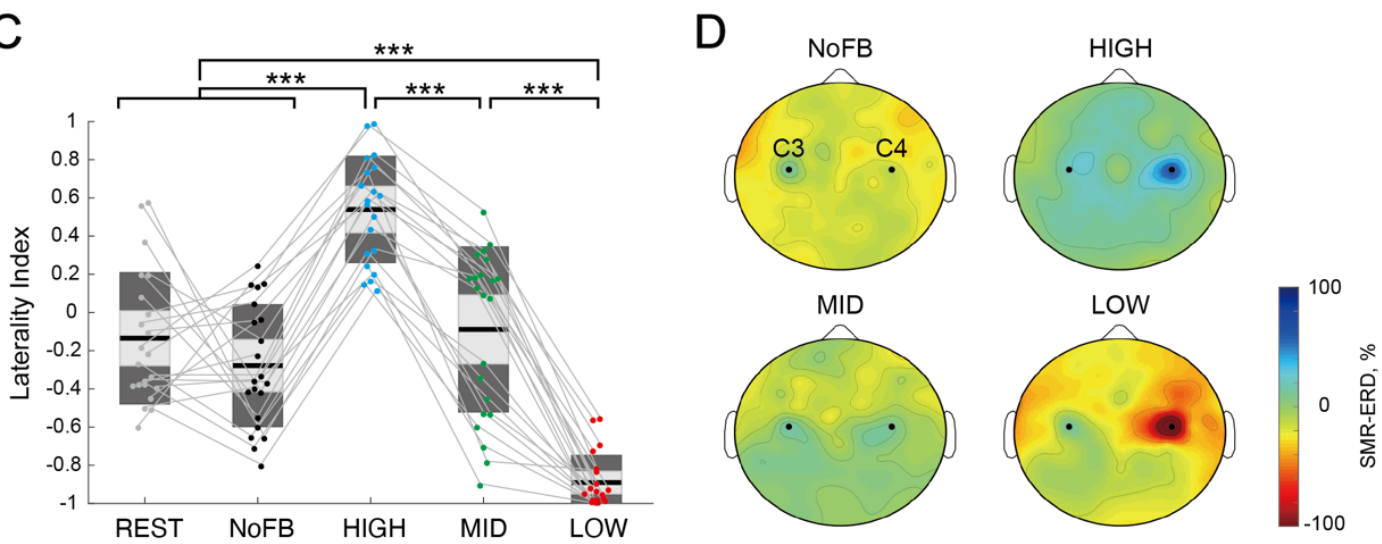

Figure 3 Target-hemisphere-specific modulation at the EEG level induced by spatially

253 bivariate BCI-based neurofeedback

254 (A), (B) Modulation effect of the contralateral and ipsilateral SMR-ERDs, respectively.

255 Individual participants are represented by colored plots and thin grey lines. The light grey box

256 represents 1.96 SEM (95\% confidence interval) and dark grey box represents 1 SD. The black

257 line indicates the group mean of the studied sample and colored plots indicate a single session.

258 Higher values represent greater sensorimotor excitability in the targeted hemisphere. The two

259 right-sided panels represent the SMR-ERD distributions during rest and MI epoch in the

260 calibration session. Based on the SMR-ERD distributions in the contralateral and ipsilateral

261 hemispheres, the target ranges of SMR-ERD during bi-EEG-triggered dual-TMS system (each 
color) were set for each participant. (C) Laterality index (LI) for each session. LI yields a value of 1 or -1 when the activity is purely ipsilateral or contralateral, respectively. Dendrograms above the bars represent the results of the post-hoc analyses. ${ }^{*} p<0.05$, ** $p<0.01$, and ${ }^{* * *} p<0.001$; all comparisons were Bonferroni corrected. (D) Spatial patterns of SMR-ERD during the MI epoch in each session (group mean). Large positive values (blue color) represent larger SMRERD (i.e., higher excitability of the SM1). The black dots represent the C3 and C4 channels.

\section{Associations between IHI magnitude and bilateral EEG patterns}

To examine the association between IHI magnitude and bilateral EEG patterns in each session, we performed a correlation analysis. In the within-subject correlation analysis between IHI magnitude and bilateral SMR-ERDs in each participant, 7 of the 22 participants showed a significant correlation between IHI magnitude and ipsilateral SMR$\operatorname{ERD}(\mathrm{r}=-0.587, p=0.003$ [representative]; $\mathrm{r}=-0.307 \pm 0.252$ [mean $\pm \mathrm{SD}$ ]), but not between IHI magnitude and contralateral SMR-ERD in three neurofeedback sessions ( $\mathrm{r}$ $=0.020, p=0.926$ [representative]; $r=-0.059 \pm 0.298$ [mean $\pm \mathrm{SD}$ ]; Figure 4A, B). In the across-subject correlation analysis between IHI magnitude and ipsilateral SMRERDs, we found a significant correlation by merging three neurofeedback sessions $(r=-$ $0.330, p=0.008$ ), but not in either session (HIGH: $\mathrm{r}=-0.217, p=0.359$, MID: $\mathrm{r}=0.037$, $p=0.869$, LOW: $\mathrm{r}=0.022, p=0.921$; Figure $4 \mathrm{C}$ ) due to inter-subject variability. The across-subject correlation analysis between IHI magnitude and contralateral SMR-ERDs showed no significant correlation by merging the three neurofeedback sessions $(\mathrm{r}=-$ $0.152, p=0.231$ ) or each session alone (HIGH: $\mathrm{r}=-0.079, p=0.739, \mathrm{MID}: \mathrm{r}=-0.092$, $p=0.685$, LOW: $\mathrm{r}=-0.200, p=0.377)$.

To analyse the associations between IHI magnitude and bilateral neural network from another perspective, interhemispheric functional connectivity during MI was examined as a key EEG signature. For the Network-intensity for the sessions (five levels: REST, NoFB, HIGH, MID, and LOW) revealed significant differences $\left(\mathrm{F}_{(4,96)}=3.04, p=0.020, \eta^{2}=0.10\right.$; REST: $0.162 \pm 0.138$; NoFB: $0.272 \pm$ 0.248; HIGH: $0.336 \pm 0.306$; MID: $0.147 \pm 0.097$; LOW: $0.232 \pm 0.205)$. A post-hoc twotailed paired t-test demonstrated significant differences between HIGH and MID sessions (difference $=-0.189$, Cohen's $d=0.83, p=0.033$; Figure 4D). Surprisingly, however, we found no significant difference between HIGH and LOW sessions (difference $=-0.104$,

294 Cohen's $d=0.40, p=1.00$; Figure 4D), suggesting that interhemispheric functional connectivity did not reflect the excitatory or inhibitory activity. 


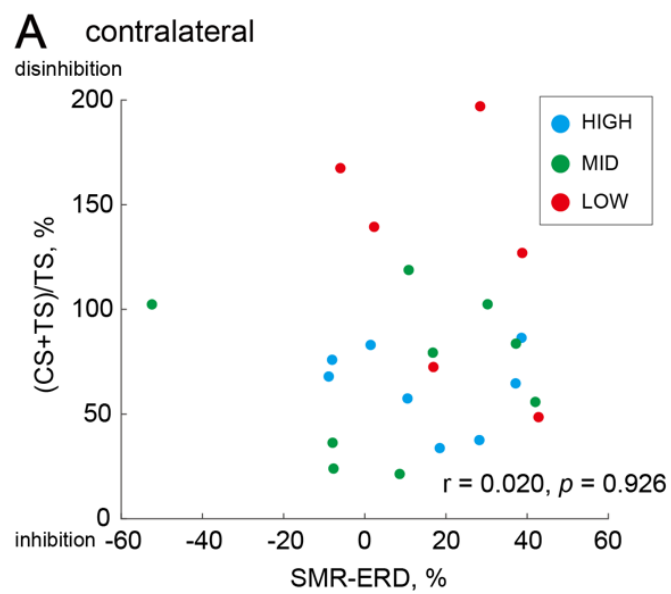

C

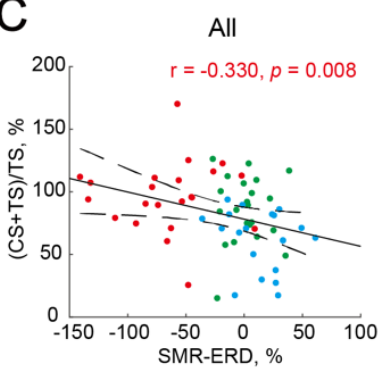

MID

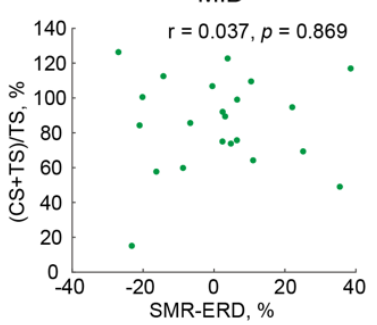

B ipsilateral

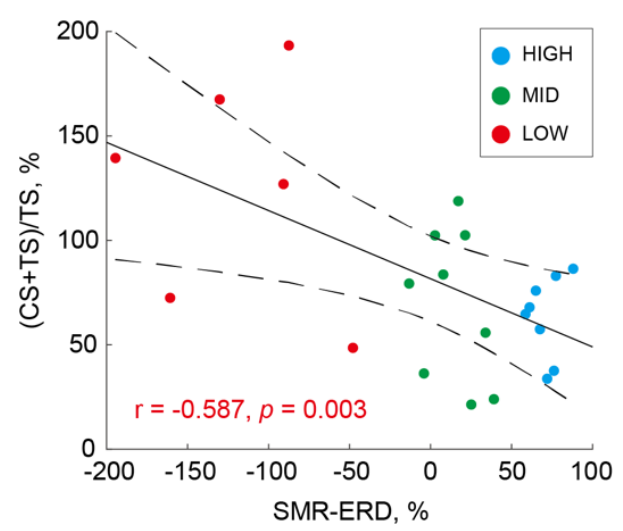

D

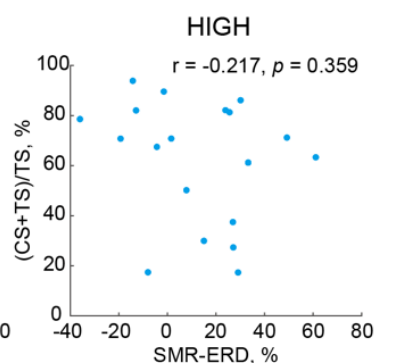

LOW

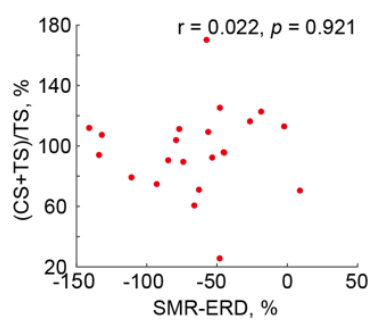

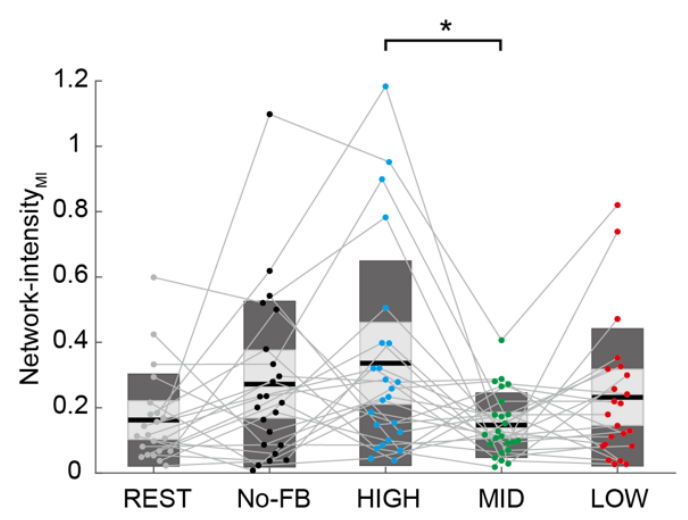

297

\section{Figure 4 Associations of IHI magnitude and bilateral EEG patterns}

299 (A), (B) Within-subject correlations between IHI magnitude and contralateral or ipsilateral SMR-

300 ERDs in a representative participant. The plot colors indicate each session, with each plot representing a single triggered trial. Solid and dotted lines represent the estimated linear regression and 95\% confidence interval, respectively. Only the ipsilateral SMR-ERD and IHI magnitude were significantly correlated; high sensorimotor excitability state in the ipsilateral hemisphere induced stronger inhibition from the ipsilateral hemisphere. (C) Across-subject correlations between IHI magnitude and ipsilateral SMR-ERDs in all three neurofeedback sessions (upper left), HIGH (upper right), MID (lower left), and LOW (lower right) sessions. Dots represent a single participant. No significant correlation was observed within either session. (D) Comparison of Network-intensity during MI across sessions. Dendrograms above the bars represent the results of the post-hoc analyses. $* p<0.05$; all comparisons were Bonferroni corrected. There was a significant difference in interhemispheric functional connectivity between

311 HIGH and MID sessions, but not between HIGH and LOW sessions, suggesting that interhemispheric functional connectivity did not reflect the excitatory or inhibitory activity. 


\section{Distinct signatures for strong versus weak manipulation of IHI}

315 Finally, we investigated the neural characteristics associated with the manipulation 316 capability of IHI $\left(\Delta \mathrm{IHI}_{\mathrm{H}-\mathrm{L}}\right)$ using correlation-based analysis (Figure $\left.5 \mathrm{~A}\right)$. In the

317 relationships between $\Delta \mathrm{IHI}_{\mathrm{H}-\mathrm{L}}$ and $\mathrm{IHI}$ in the REST session ( $\mathrm{IHI}_{\text {rest }}$ ), we found a 318 significant correlation between $\Delta \mathrm{IHI}_{\mathrm{H}-\mathrm{L}}$ and $\mathrm{IHI}_{\mathrm{rest}}(\mathrm{r}=-0.447, p=0.044)$, indicating that 319 participants with greater IHI at rest were able to strongly manipulate the IHI (Figure 5B).

320 For the relationships between resting-state effective inhibitory interhemispheric network 321 assessed by $\mathrm{IHI}_{\text {rest }}$ and interhemispheric functional connectivity at the EEG level

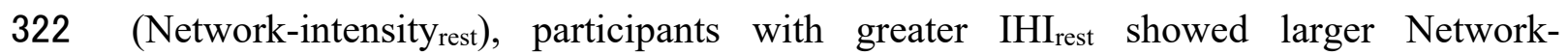
323 intensityrest $(\mathrm{r}=0.547, p=0.013$; Figure 5C). Connectivity results in the other frequency 324 bands are shown in Supplementary files ("Connectivity results in the other frequency 325 bands"). Furthermore, we verified whether $\mathrm{IHI}_{\text {rest }}$ may be associated with intrinsic EEG 326 profiles in the NoFB session including bilateral SMR-ERDs and LI during MI. We found 327 a significant correlation between $\mathrm{IHI}_{\text {rest }}$ and ipsilateral SMR-ERD during MI $(\mathrm{r}=-0.619$, $328 p=0.004)$, but not between $\mathrm{IHI}_{\mathrm{rest}}$ and contralateral SMR-ERD during $\mathrm{MI}(\mathrm{r}=-0.283, p$

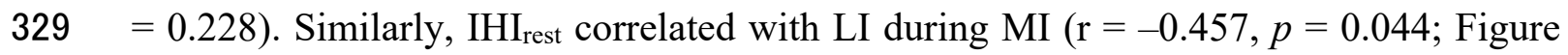
330 5D). 
A

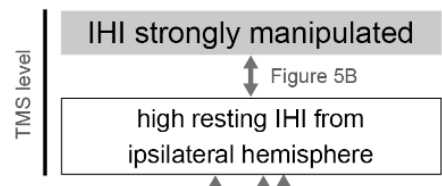

Figure 5C ₹ 4 T Figure 5D

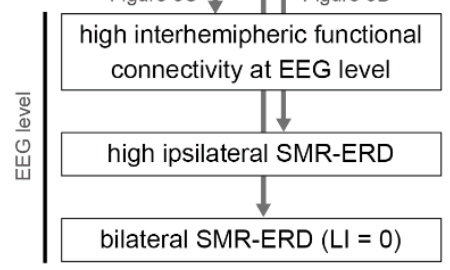

C

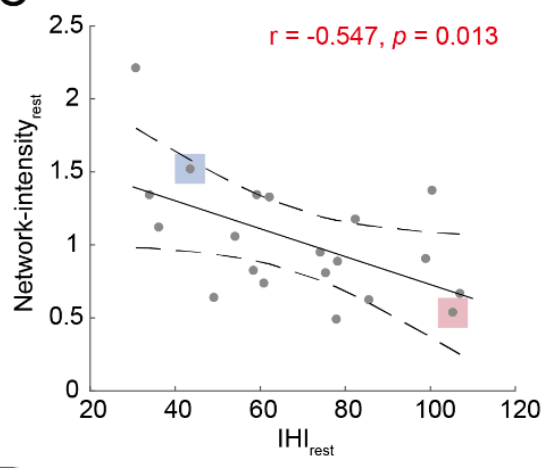

B

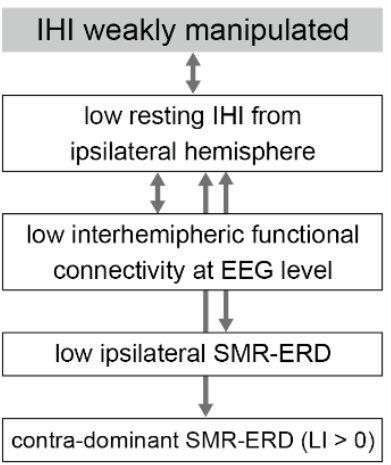

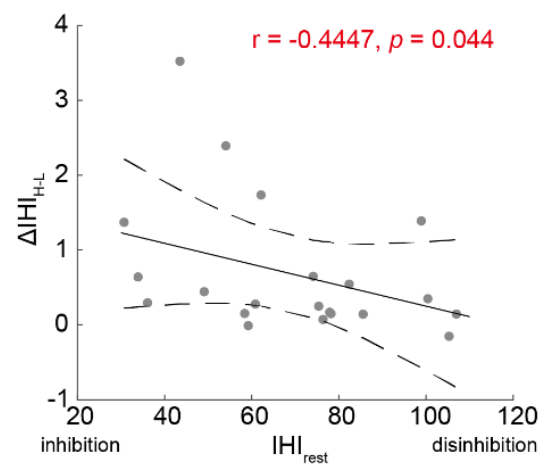

D
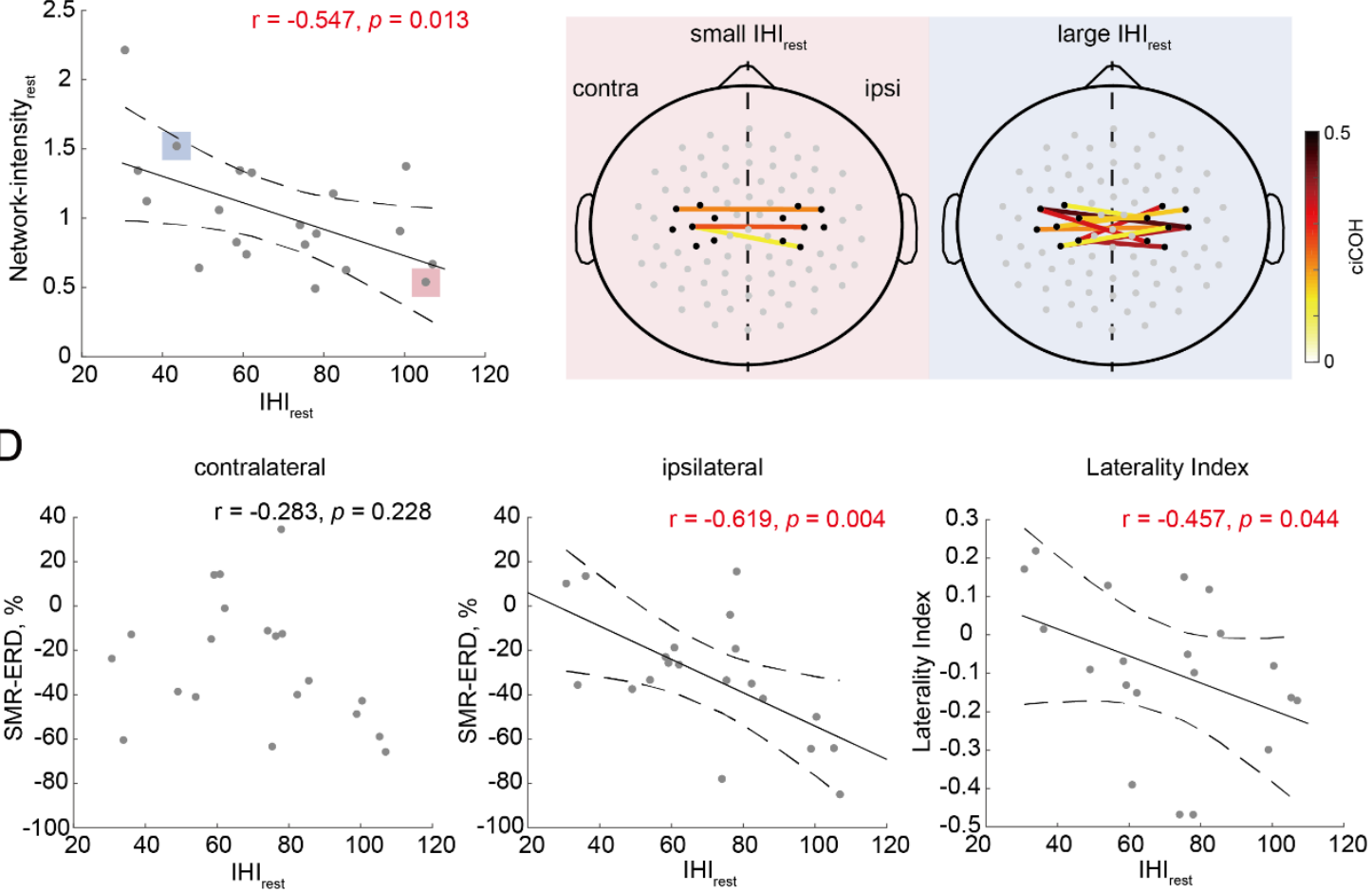

332 Figure 5 Neural characteristics depending on the manipulation capability of IHI

333 (A) Overview of the relationships between biomarkers from EEG and TMS data to probe the distinct signatures for strong versus weak manipulation of IHI. Arrows corresponds to a single panel. (B) Across-subject correlations between the manipulation capability of IHI and intrinsic IHI magnitude at rest. SMR-ERDs in a representative participant. Dots represent a single participant. Solid and dotted lines represent the estimated linear regression and $95 \%$ confidence interval, respectively. Participants with greater IHI at rest were able to strongly manipulate IHI. (C) Across-subject correlations between IHI at rest and EEG profiles showed significant correlations between $\mathrm{IHI}_{\text {rest }}$ and ipsilateral SMR-ERD and LI, but not between $\mathrm{IHI}_{\mathrm{rest}}$ and contralateral SMR-ERD. (D) The left-sided panel shows across-subject correlations between IHI at rest and resting-state interhemispheric functional connectivity. The two right-sided panels indicate the significant interhemispheric connections ("Connectivity Analysis" in Methods) of the two representative participants with small and large $\mathrm{IHI}_{\mathrm{rest}}$, respectively. The solid lines 
indicate a significant connection, and large positive values (dark red color) represent strong connections. The black dots around bilateral SM1 denote the seed channels, $\mathrm{C} 3$ or $\mathrm{C} 4$, and six neighboring channels. The gray dots represent other EEG channels.

\section{Discussion}

In the present study, we aimed to determine whether it is possible to consciously selfmanipulate the IHI magnitude and uncover related neural activity behind IHI, while controlling bilateral SMR-ERDs via closed-loop spatially bivariate BCI-based neurofeedback. This was the first study to show voluntary IHI-state manipulation with a large dynamic range related to variation in ipsilateral, rather than contralateral, sensorimotor excitability expressed by SMR-ERD and a resting-state interhemispheric network at the TMS (IHI rest) and EEG (interhemispheric Network-intensityrest) levels, which was accompanied by a balance of SMR-ERDs over bilateral SM1s.

Previous neurofeedback studies have demonstrated that it is possible to gain voluntary control over central nervous system activity without externally administered interventions, if appropriate neurofeedback is embedded in a reinforcement learning task, such as food rewards for animals (Engelhard et al., 2013; Fetz, 2013) and visually rewarding stimuli for humans (Thompson et al., 2009). However, conventional BCIbased neurofeedback of the SMR signal from one hemisphere does not guarantee spatial specific activation of the sensorimotor activity in the targeted hemisphere (Buch et al., 2008; Caria et al., 2011; Soekadar et al., 2015a) because sensorimotor activities in the left and right hemispheres potentially influence one another, making pathway-specific IHI manipulation difficult. Using spatially bivariate BCI-based neurofeedback enables participants to volitionally increase or decrease (bidirectional) the ipsilateral sensorimotor excitability, while maintaining constant contralateral sensorimotor excitability. Ipsilateral SMR-ERD (but not contralateral), reflecting IHI magnitude (Figure 3B) and their significant correlation (Figure 4C), were compatible with previous studies (Haegens et al., 2011; Madsen et al., 2019; Sauseng et al., 2009; Takemi et al., 2013; Thies et al., 2018; Zarkowski et al., 2006). The in vivo cortical recordings in monkeys revealed that pericentral alpha power was inversely related with the normalized firing rate in the sensorimotor regions (Haegens et al., 2011). In humans, several studies using single-pulse TMS of the motor hand area combined with EEG tested how ongoing pericentral oscillatory activity impacts corticomotor excitability reflected by the MEP amplitude. These EEG-TMS studies found a negative linear relationship between pre-stimulus SMR- 
379 ERD and MEP amplitude through both offline (Sauseng et al., 2009; Takemi et al., 2013; 380 Zarkowski et al., 2006) and online (Madsen et al., 2019; Thies et al., 2018) approaches.

381 As for IHI through the transcallosal fiber, it is predominantly regulated through direct 382 postsynaptic mechanisms in the apical dendritic shafts of pyramidal neurons and a 383 specific cortical microcircuitry mediated by dendritic $\mathrm{GABA}_{\mathrm{B}}$ receptors in inhibitory 384 interneurons (Palmer et al., 2012). Consistent with this phenomenon, down-regulation of 385 ipsilateral SMR-ERD, for example, may influence the excitation of the ipsilateral 386 transcallosal pyramidal neuron followed by disinhibition of the contralateral inhibitory 387 interneurons during unilateral upper limb MI. Therefore, pronounced changes in cortical 388 mechanism despite the absence of sensory input and constant MEP amplitude elicited by 389 single TS suggest that the increase or decrease of IHI magnitude was at least partly of 390 cortical, rather than spinal, origin. These neural mechanisms can be explored further by using triple pulse procedures ( $\mathrm{Ni}$ et al., 2011) in which disinhibition can be directly measured as the reduction of short intracortical inhibition.

The potential contribution of resting-state IHI magnitude and interhemispheric functional connectivity in the alpha and high beta bands to the current results of manipulation capability in IHI (Figure 5B, C, and Supplementary figure 5C) can be speculated by considering the previous studies. Interregional communication is accompanied by synchronized oscillations in different brain regions (Fries, 2005; Varela et al., 2001), and this synchronization can be evaluated by functional connectivity. Because both hemispheres are structurally connected by transcallosal projection and exhibit functional cross talks (Hofer and Frahm, 2006; Meyer et al., 1995), the manipulation capability of IHI suggests it is associated with a structural connectivity (assessed by paired-pulse TMS and intrinsic EEG synchronization between hemispheres) and local oscillatory power entrainment. As for frequency band, it is well known that the intensity of SMR-ERD in the beta band reflects the sensorimotor cortical excitability and cortico-muscular activation (Hussain et al., 2019; Schulz et al., 2014). This multimodal EEG-TMS approach proves that the bilateral alpha and beta activities observed in the current study served to regulate the inhibition and facilitation of inhibitory functional coupling of interhemispheric interaction over motor cortices.

In addition, real-time fMRI-based neurofeedback to a single region of interest

410 (Sitaram et al., 2017; Weiskopf et al., 2004) or interregional functional connectivity (Liew 411 et al., 2016; Pereira et al., 2019) can be used for volitional self-modulation of neuronal 412 connectivity and could serve as a possible therapeutic tool for motor or cognitive training 
413 in diseases related to impaired interregional interaction. However, even fMRI-based

414 functional connectivity neurofeedback with high spatial resolution, based on blood

415 oxygenation level-dependent signal, cannot differentiate between excitatory and

416 inhibitory activities between these regions. Indeed, our results indicated no significant

417 difference in Network-intensity during MI between HIGH and LOW sessions (Figure

418 4D). This interesting result would be explained by the following hypothesis: In LOW

419 session, for example, down-regulation of the ipsilateral excitability leads to temporal

420 down-regulation of the contralateral excitability since both hemispheres influence each

421 other, which results in synchronized interhemispheric oscillatory activities; however, the

422 contralateral excitability remains at the baseline level due to BCI-based neurofeedback

423 and the interhemispheric input from the ipsilateral hemisphere increases simultaneously,

424 which affect the down-regulation of intracortical inhibition in the contralateral

425 hemisphere (Takemi et al., 2013), while maintaining constant right finger MEP. In

426 addition, disinhibition of the inhibitory interneurons in the contralateral hemisphere

427 projecting transcallosal pyramidal neuron causes increased IHI to the ipsilateral

428 hemisphere, leading to similar sum of bidirectional IHI between HIGH and LOW

429 sessions. Therefore, functional connectivity does not change. Hence, our results provided

430 evidence that interhemispheric functional connectivity using fMRI cannot distinguish

431 between excitatory and inhibitory neural activities, and ensured that our approach is

432 useful as a direct IHI manipulator.

433 Interhemispheric activity is also passively modulated by externally administered

434 interventions, for example, tDCS or rTMS over the motor cortices (Gilio et al., 2003;

435 Peña-Gómez et al., 2012; Williams et al., 2010). Although such tools have

436 neuromodulation efficacy, their long-term sustained effects are often limited and they

437 result in local and remote effects without spatial specificity (Di Pino et al., 2014; Notturno

438 et al., 2014; Weiskopf et al., 2004). Conversely, under the conscious self-learning

439 environment, volitional control over MEP amplitudes is retained for at least 6 months

440 without further training (Ruddy et al., 2018), which supports the prediction of long-term

441 efficacy and safety of pathway-specific IHI self-manipulation. Furthermore, the effect

442 size of IHI manipulation in the current study (Cohen's $d=1.50$ ) was approximately 1-2-

443 fold higher than that of representative tDCS (Cohen's $d=1.55$ ) (Williams et al., 2010)

444 and rTMS studies (Gilio et al., 2003) (Cohen's $d=0.80$; note that it is a read from the

445 graph). Based on a previous study of neurofeedback training combined with an external

446 administered interventions (Ang et al., 2012), their combination may be useful for 
447

448

449

450

451

452

453

454

455

456

457

458

459

460

461

462

463

464

465

466

467

468

469

470

471

472

473

474

475

476

477

478

479

480

facilitating neural plasticity.

Our evidence and techniques are expected to be applied in various fields, for example, in the context of neurorehabilitation. In stroke patients, the interhemispheric imbalance model predicts the presence of asymmetry in the interhemispheric sensorimotor network, with excessive inhibition from the non-affected hemisphere limiting maximal recovery (Murase et al., 2004). Therefore, guiding effective inhibitory interhemispheric network represented by IHI to the appropriate pattern through both targeted up-conditioning in the affected hemisphere and down-conditioning in the nonaffected hemisphere may contribute to reduced abnormal IHI and enhanced achievable functional recovery (Chieffo et al., 2013; Di Pino et al., 2014; Dong et al., 2006; Hummel and Cohen, 2006). Although the rehabilitation strategy of attempting to rebalance interhemispheric interactions in order to improve motor recovery after stroke is controversial (Bundy et al., 2017; Xu et al., 2019), the current technique can be tailored either to upregulate the damaged hemisphere, down-regulate the intact hemisphere, a combination of both, or vice versa depending on the patient's specific needs. Current proof-of-concept data reveal for the first time that self-manipulation of IHI via BCI-based neurofeedback is equal or superior to the conventional tools. Future work probing the residual ability to manipulate IHI in stroke patients is warranted.

In conclusion, we presented an innovative approach to voluntarily and bidirectionally manipulate the state of IHI, by directly targeting SMR-ERDs in a spatially bivariate BCI-based neurofeedback paradigm. This approach provides the opportunity to understand the inhibitory sensorimotor functions and paves the way for new technologies that allow the user/patient to regulate aspects of their brain function to reach the desired states, e.g., for neurorehabilitation and enhanced motor performance.

\section{Materials and Methods}

\section{Study design}

The current study was performed in accordance with approved guidelines and regulations, such as the CONSORT Statement (Moher et al., 2001) and CRED-nf checklist (Ros et al., 2020). The experiment consisted of five sessions: (1) resting-state (REST); (2) right finger MI without visual feedback (NoFB), (3) high (HIGH); (4) middle (MID); and (5) low excitability states of the ipsilateral SM1 during BCI-based neurofeedback (LOW) (details in "Experimental sessions"). The difference of IHI magnitude in the last three sessions (i.e., HIGH, MID, and LOW sessions) was the primary dependent variable of 
481

482

483

484

485

486

487

488

489

490

491

492

493

494

495

496

497

498

499

500

501

502

503

504

505

506

507

508

509

510

511

512

513

514

interest. REST and NoFB sessions were treated to estimate individual baseline during rest and MI for the offline analysis.

To estimate the appropriate sample size for this study, a preliminary experiment was conducted before the main experiment. In the preliminary experiment, four healthy participants (not included in the main experiment) performed BCI-based neurofeedback training and underwent brain state-dependent dual-coil brain stimulation, similar to the main experiment. We calculated the IHI magnitude in each session. Then, an a priori power analysis $(\alpha=0.05,1-\beta=0.8$, two-sided tests, Bonferroni corrected) focusing on the IHI magnitude using the statistical package G*Power 3.1 (Faul et al., 2009) was conducted. Because the preliminary experiment showed a large effect size on the IHI differences between HIGH $(65.0 \pm 22.8$, mean \pm SD) and MID $(90.7 \pm 23.4)$ sessions (Cohen's $d=1.12)$, and between MID (90.7 \pm 23.4$)$ and LOW (106.3 \pm 13.1$)$ sessions (Cohen's $d=0.82$ ), we calculated that 24 participants were needed (Cohen, 2013, 1992).

\section{Participants}

Twenty-four volunteers ( 2 females and 22 males; mean age \pm SD: $23.4 \pm 2.0$ years; age range: 21-27 years) participated in this study. All participants had normal or correctedto-normal vision and reported no history of neurological or psychological disorders. All participants were right-handed (Laterality Quotient: $72.2 \pm 30.9 \%$ ) as assessed by the Edinburgh Inventory (Oldfield, 1971). Participants were excluded if the resting motor threshold (RMT) of the right and left first dorsal interosseous (FDI) muscles was $\leq 70 \%$ of the maximum stimulator output (MSO) during the TMS experiment. This criterion ensured that the TMS stimulator would be able to perform at the required intensities for the whole duration of the experiment (Stefanou et al., 2018; Zrenner et al., 2018). We did not exclude participants based on the EEG characteristics such as the magnitude of their endogenous SMR activity (Madsen et al., 2019; Safeldt et al., 2017), to verify our hypothesis in a Proof-of-Concept study. Twenty-two participants ( 2 females and 20 males; mean age \pm SD: $23.3 \pm 1.9$ years; age range: $21-27$ years; Laterality Quotient: $72.5 \pm 32.2 \%$ ) attended the ensuing neurofeedback training with brain stimulation. The results presented in the EEG part are from all 24 participants, while IHI results are from the 22 participants that completed the whole experiment. Four of the 120 sessions from four participants (two REST, one NoFB, and one HIGH session) were excluded due to corrupted data.

The experiments conformed to the Declaration of Helsinki and were performed in 
515 accordance with the current TMS safety guidelines of the International Federation of

516 Clinical Neurophysiology (Rossi et al., 2009). The experimental procedure was approved

517 by the Ethics Committee of the Faculty of Science and Technology, Keio University (no.:

518 31-89, 2020-38, and 2021-74). Written informed consent was obtained from participants

519 prior to the experiments.

520

\section{EEG/EMG data acquisition}

522 EEG signals were acquired using a 128-channel Hydrogel Geodesic Sensor Net 130 523 system (Electrical Geodesics Incorporated [EGI], Eugene, OR, USA) in a quiet room.

524 EEG data were collected at a sampling rate of $1 \mathrm{kHz}$ and transmitted via an Ethernet 525 switch (Gigabit Web Smart Switch; Black Box, Pennsylvania, USA) to the EEG 526 recording software (Net Station 5.2; EGI and MATLAB R2019a; The Mathworks, Inc, 527 Massachusetts, USA). The ground and reference channels were placed at $\mathrm{CPz}$ and $\mathrm{Cz}$, 528 respectively. The impedance of all channels, excluding the outermost part, was 529 maintained below $30 \mathrm{k} \Omega$ throughout the experiment to standardize the EEG recordings 530 (Ferree et al., 2001). This impedance standard was consistent with other studies using the same EEG system (Carter Leno et al., 2018b, 2018a; Robertson et al., 2019).

Surface EMGs were recorded from the FDI and ADM of the left and right hands using two pairs of $\mathrm{Ag} / \mathrm{AgCl}$ electrodes $(\phi=10 \mathrm{~mm})$ in a belly-tendon montage.

534 Impedance for all channels was maintained below $20 \mathrm{k} \Omega$ throughout the experiment.

535 EMG signals were digitized at $10 \mathrm{kHz}$ using Neuropack MEB-2306 (Nihon Kohden, 536 Tokyo, Japan). The EMG data from each trial were stored for offline analysis on a computer from $500 \mathrm{~ms}$ before to $500 \mathrm{~ms}$ after the TMS pulse. Simultaneously, 5-10 ms of data were transferred immediately after collection to a computer for real-time analysis.

539 In case of muscular contraction due to finger movement, the experimenter reminded 540 participants to relax their muscles and ensure absence of muscle activity during MI. To 541 monitor the real-time surface EMG signals, EMG signals were band-pass filtered (5$5421000 \mathrm{~Hz}$ with 2nd order Butterworth) with a 50-Hz notch to avoid power-line noise 543 contamination; the root mean square of the filtered EMG signal from the FDI for the 544 previous $1000 \mathrm{~ms}$ of data was displayed on the second experimenter's screen in the form 545 of a bar.

546 Throughout the experiment, the participants were seated in a comfortable chair 547 with stable forearm support and performed unilateral right index finger abduction MI.

548 The wrist and elbow joint angles were fixed to a neutral posture at the armrest. The 
549

550

551

552

553

554

555

556

557

558

559

560

561

562

563

564

565

566

567

568

569

570

571

572

573

574

575

576

577

578

579

580

581

582

participants were instructed to maintain this posture and were visually monitored by the experimenter throughout the EEG and MEP measurements. During MI, the forelimbs were placed in a prone position, with natural elbow and shoulder joint angles to help prevent the muscle activity.

\section{TMS protocol}

For the TMS experiments, TMS was delivered using two interconnected single-pulse magnetic stimulators (The Magstim BiStim²; Magstim, Whiteland, UK) producing two monophasic current waveforms in a 70-mm figure-of-eight coil. We identified the optimal left and right coil positions over the hand representation area at which a singlepulse TMS evoked a MEP response in the FDI muscle with the lowest stimulus intensity, referred to as the motor hotspot. The TS was delivered to the motor hotspot of the right M1, with the handle of the coil pointing backward and approximately $45^{\circ}$ to the midsagittal line. The other coil for the CS was similarly placed over the motor hotspot of the left M1 but slightly reoriented at $45-60^{\circ}$ relative to the midsagittal line because it was not possible to place two coils in some participants with small head size. This orientation is often chosen in IHI studies (Daskalakis et al., 2002) since it induces a posterior-anterior current flow approximately perpendicular to the anterior wall of the central sulcus, which evokes MEPs at the lowest stimulus intensities (Rossini et al., 2015). To immobilize the head and maintain fixed coil positions over the motor hotspots during the experiment, chin support and coil fixation arms were used. The position of the TMS coil was monitored using the Brainsight TMS navigation system (Rogue Research, Cardiff, UK), so that the optimal coil orientation and location remained constant throughout the experiment.

RMT was defined as the lowest stimulator output eliciting an MEP in the contralateral side of relaxed FDI of $>50 \mu \mathrm{V}$ peak-to-peak in 5 out of 10 consecutive trials (Groppa et al., 2012; Rossini et al., 1994). The stimulus intensities of the left and right M1 to evoke MEP of $1 \mathrm{mV}$ peak-to-peak amplitude from the relaxed right and left FDIs $\left(\mathrm{SI}_{1 \mathrm{mV}}\right)$ were also determined for the following dual-coil paired-pulse TMS. The average $\mathrm{SI}_{1 \mathrm{mV}}$ of TS and CS were $124 \pm 11 \%$ RMT and $132 \pm 13 \%$ RMT, respectively.

\section{IHI evaluation}

IHI from the ipsilateral (right) to the contralateral (left) M1 was probed using a dual-coil paired-pulse TMS paradigm. CS was applied to the right M1, followed a few milliseconds 
583 later by a TS delivered to the left M1 (Ferbert et al., 1992). Due to the time constraint, 584 the ISI in the present study was uniformly set to $10 \mathrm{~ms}$, in accordance with previous 585 studies (Duque et al., 2005; Harris-Love et al., 2007; Murase et al., 2004; Tsutsumi et al., 586 2012); however, an ideal ISI would vary across individuals. Additionally, in a preliminary 587 experiment with four participants, it was confirmed that IHI was clearly observed when 588 ISI was set to $10 \mathrm{~ms}$. The stimulus intensity remained constant throughout the experiment 589 for each participant.

590 To validate IHI measurement under bi-EEG-triggered dual-TMS setup, IHI 591 curves were obtained in 20 of 24 participants prior to the main experiment, where a CS 592 of varying intensity (five different intensities, 100-140\% of RMT, in steps of 10\% RMT) 593 preceded the TS. Ten conditioned MEPs were collected for each CS intensity, along with 59410 unconditioned MEPs (i.e., TS was given alone) in randomized order. The peak-to595 peak amplitudes of the conditioned MEPs were averaged for the different CS intensities

596 and expressed as a percentage of the mean unconditioned MEP amplitude. IHI intensity 597 curves (Figure 2) ensured that IHI was approximately half-maximum for each participant 598 when $120-130 \%$ RMT of CS intensity was applied, similar to previous EEG-TMS experiment (Stefanou et al., 2018; Tsutsumi et al., 2012).

600

\section{Spatially bivariate BCI-based neurofeedback}

602 The present study was conducted based on a spatially bivariate BCI-based neurofeedback 603 that displays bi-hemispheric sensorimotor cortical activities, which we recently 604 developed in our laboratory (Hayashi et al., 2021, 2020). This method allows participants 605 to learn to regulate these two variates at the same time and induce changes in target606 hemisphere-specific SMR-ERD. Visual feedback was provided on a computer screen in 607 the form of cursor movements in a two-dimensional coordinate, in which $\mathrm{x}$ and $\mathrm{y}$ axis 608 corresponded to the degree of the ipsilateral and contralateral SMR-ERD, respectively. 609 The axis range was set from the 5th (i.e., SMR-ERS) to 95th (i.e., SMR-ERD) percentile 610 of intrinsic SMR-ERD distribution in EEG calibration session, and the origin-position ( $\mathrm{x}$ $611=0, \mathrm{y}=0)$ represented median values of bilateral SMR-ERDs. The cursors were presented 612 at the origin-position at the initiation of a trial, and values exceeding the boundary were 613 rounded to the 5 th or 95 th percentile. A key point of the current methodology is that, for 614 example, when participants were instructed to move the cursor toward the middle right $615(\mathrm{x}>0, \mathrm{y}=0)$ in the two-dimensional coordinate, the position showed a strong SMR-ERD 616 in the ipsilateral hemisphere and moderate SMR-ERD in the contralateral hemisphere. 
617 Therefore, spatially bivariate BCI-based neurofeedback enables us to investigate how 618 sensorimotor excitability in the target hemisphere (i.e., ipsilateral side) contributes to IHI 619 while maintaining constant contralateral sensorimotor excitability.

620 During MI, participants were asked to perform a right index finger kinesthetic MI 621 from the first-person perspective with equal time constants of $0.5 \mathrm{~Hz}$ cycle. Kinesthetic 622 MI was performed because a previous study demonstrated that the focus of EEG activity 623 during kinesthetic MI was close to the sensorimotor hand area, whereas visual MI did not 624 reveal a clear spatial pattern (Neuper et al., 2005). To improve MI task compliance (i.e., 625 whether all participants successfully performed the MI in the same manner), we not only 626 asked them to perform kinesthetic MI from a first-person perspective, but also asked them 627 to perform a rehearsal before each session. In addition, we confirmed that SMR was 628 observed in a frequency-specific, spatiotemporal-specific, and task-related manner 629 through offline analysis after each session. These characteristics of SMR-ERD indicate 630 that kinesthetic MI, not visual MI, was performed correctly (Neuper et al., 2005; 631 Pfurtscheller and Neuper, 1997).

632

\section{Real-time brain state-dependent dual-coil brain stimulation}

634 The bi-EEG-triggered dual-TMS setup uses an online output of the raw EEG signal and 635 analyzes it in real time to trigger TMS pulses depending on the instantaneous bilateral 636 spatially filtered SMR-ERD of the recorded EEG. The real-time SMR-ERD intensity in 637 each hemisphere (relative to the average power of the 1-5 s of the resting epoch) was 638 obtained every $100 \mathrm{~ms}$ and calculated using the last 1-s data as follows (Hayashi et al., 639 2020): (1) acquired raw EEG signals recorded over SM1 underwent a 1-70-Hz second640 order Butterworth bandpass filter and a 50-Hz notch filter; (2) filtered EEG signals were 641 spatially filtered with a large Laplacian (60 $\mathrm{mm}$ to set of surrounding channels), which 642 subtracted the average value of the surrounding six channel montage from that of the 643 channel of interest (i.e., C3 and C4, respectively). This method enabled us to extract the 644 task-related EEG signature and improve the signal-to-noise ratio of SMR signals 645 (McFarland et al., 1997; Tsuchimoto et al., 2021). In addition, the large Laplacian method 646 is better matched to the topographical extent of the EEG control signal than the small 647 Laplacian and ear reference methods (McFarland et al., 1997); (3) a fast Fourier transform 648 was applied to the spatially large Laplacian filtered EEG signals; (4) the power spectrum 649 was calculated by calculating the square of the Fourier spectrum; (5) the alpha band power 650 was obtained by averaging the power spectrum across the predefined alpha target 
651 frequencies from the EEG calibration session (described below); (6) the alpha band power

652

653

654

655

656

657

658

659

660

661

662

663

664

665

666

667

668

669

670

671

672

673

674

675

676

677

678

679

680

681

682

683

684

was time-smoothed by averaging across the last five windows (i.e., $500 \mathrm{~ms}$ ) to extract the low-frequency component for high controllability. The slow fluctuation component is beneficial to neurofeedback training because it reduced the flickering and improved the signal-to-noise ratio of the SMR signal (He et al., 2020; Kober et al., 2018); and (7) SMRERD was obtained by calculating the relative power to the average power during the resting epoch. SMR-ERDs of the last ten segments were displayed and updated every 100 $\mathrm{ms}$, allowing participants to compare the current and past conditions. Thereafter, a cue signal was generated to trigger the magnetic stimulator of CS stimulus when the signal reached the predetermined target that the SMR-ERD threshold was exceeded and transmitted Transistor-Transistor Logic pulse to the magnetic stimulator of TS stimulus $10 \mathrm{~ms}$ later by Neuropack MEB-2306 system.

\section{Experimental sessions}

Before the main IHI experiment, maximal voluntary contraction (MVC) was measured (Figure 6A). Full-length isometric abduction of the right and left index and little fingers were performed once after several exercises; each execution lasted $5 \mathrm{~s}$ with a 30 -s rest between contractions to allow for recovery from mental fatigue. Each MVC was obtained by calculating the root mean square of stable $3 \mathrm{~s}$ of filtered EMG data.

Next, to determine the parameters of the bi-EEG-triggered dual-TMS setup, an EEG calibration session consisting of 20 trials, providing real-time SMR-ERD only on the contralateral side, was performed for each participant prior to the IHI experiment. Each trial was initiated by a 5-s resting epoch, followed by a 1-s ready epoch, and completed by a 6-s MI epoch. During this 12-s trial period, participants were asked not to move, blink, or swallow to prevent EEG artefacts derived from non-neural activity. After each 12-s trial, the screen went black for $3 \mathrm{~s}$ (Figure 6A). Participants were allowed to move freely to avoid mental fatigue during this interval period, before the next trial started. Thereafter, the target frequencies in the contralateral and ipsilateral SM1 were determined for each participant in order to feedback the most reactive frequency. Since SMR-ERD in the alpha band is a reliable EEG biomarker of increased neuronal excitability in SM1, corticospinal tract, and thalamocortical systems (Neuper et al., 2006; Soekadar et al., 2015b; Takemi et al., 2018, 2015, 2013; Yuan et al., 2010), the target frequencies were selected from the alpha band $(8-13 \mathrm{~Hz})$ by calculating the mean intensity of SMR-ERD with a $3-\mathrm{Hz}$ sliding bin and $2-\mathrm{Hz}$ overlap. Second, the target 
685

686

687

688

689

690

691

692

693

694

695

696

697

698

699

700

701

702

703

704

705

706

707

708

709

710

711

712

713

714

715

716

717

718

ranges of SMR-ERD during bi-EEG-triggered dual-TMS setting were normalized for each participant based on SMR-ERD distribution in the contralateral and ipsilateral hemispheres.

After the calibration session, the main IHI experiment with dual-coil paired-pulse TMS was performed in five consecutive sessions (10-min each) with fixed CS and TS intensities. Each session consisted of 40 trials and the task sequence of each trial was similar to that of the EEG calibration session. Five experimental sessions comprised different conditions as follows: (1) resting-state where participants were instructed to relax and look at the origin of the 2-D coordinates on the computer screen in front of them (REST); (2) right finger MI without visual feedback (NoFB); participants tried to achieve (3) high (HIGH); (4) middle (MID), and (5) low excitability states of the ipsilateral SM1 during BCI-base neurofeedback (LOW). The last three HIGH, MID, and LOW sessions were arranged in a random order, and in these sessions, participants received visual feedback based on the SMR-ERDs from both contralateral and ipsilateral hemispheres.

In each session, five trigger conditions were tested, with approximately equal numbers of paired pulses and unconditioned test pulses. EEG-triggered TMS timing ranged from 0.5 to $5.5 \mathrm{~s}$ during the MI epoch, and non-triggered TMS (referred to as the failed trial) was delivered in shuffled timing ranging from 5.5 to $6 \mathrm{~s}$ during the MI epoch to see the influence of spontaneous SMR fluctuations on IHI. Trigger conditions were determined based on the intrinsic sensorimotor cortical activity of each participant and was calculated in the EEG calibration session. The predetermined target ranges of SMRERD was expressed by a blue rectangle on the computer screen in each session are as follows (Figure 6B): (1) 37.5-62.5th percentile of SMR-ERD distribution during rest in both hemispheres (REST session); (2) 37.5-62.5th percentile of SMR-ERD distribution during $\mathrm{MI}$ in the contralateral hemisphere and 5-95th percentile of SMR-ERD distribution during MI in the ipsilateral hemisphere (NoFB session); (3) 37.5-62.5th percentile of SMR-ERD distribution during MI in the contralateral hemisphere and 7595th percentile of SMR-ERD distribution during MI in the ipsilateral hemisphere (HIGH session); (4) 37.5-62.5th percentile of SMR-ERD distribution during MI in the contralateral hemisphere and 37.5-62.5th percentile of SMR-ERD distribution during MI in the ipsilateral hemisphere (MID session); and (5) 37.5-62.5th percentile of SMR-ERD distribution during $\mathrm{MI}$ in the contralateral hemisphere and 5-25th percentile of SMRERD distribution during MI in the ipsilateral hemisphere (LOW session). The last three sessions (i.e., HIGH, MID, and LOW sessions) were our primary dependent variables of 
719 interest to verify how SMR-ERD in the ipsilateral hemisphere contributes to IHI while 720 maintaining constant contralateral SMR-ERD. The REST and NoFB sessions served as 721 controls to determine the intrinsic IHI magnitude without a neurofeedback paradigm. To 722 evaluate the difficulty of each neurofeedback session, the mean of the sum of the success 723 triggered trials $( \pm 1 \mathrm{SD}$ ) of all sessions (except NoFB session because it has a wide 724 triggered range) was calculated. The waiting time for a triggered event from MI onset 725 was measured for each session.

A
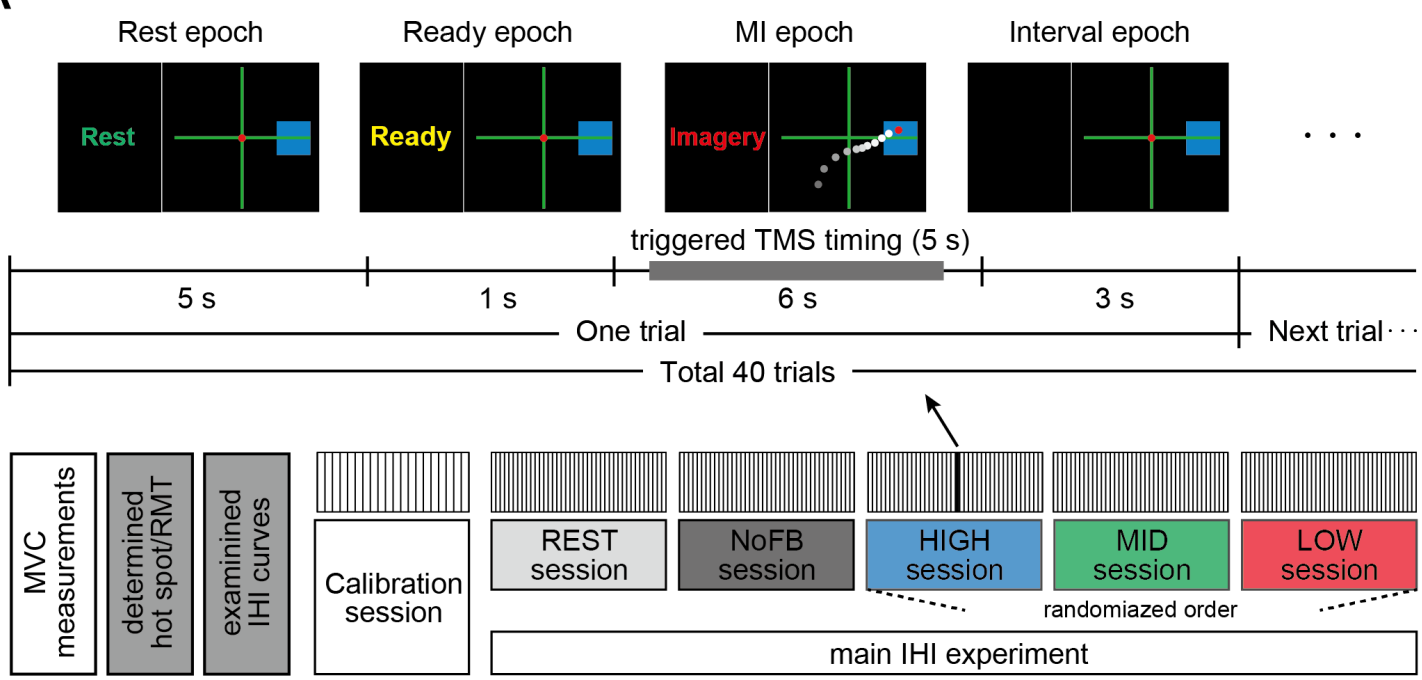

B

\begin{tabular}{ccc} 
Session & Contralateral SMR-ERD (percentile) & Ipsilateral SMR-ERD (percentile) \\
\hline REST & 37.5-62.5th (rest) & 37.5-62.5th (rest) \\
\hline NoFB & 37.5-62.5th (motor imagery) & 5-95th (motor imagery) \\
\hline HIGH & 37.5-62.5th (motor imagery) & 75-95th (motor imagery) \\
\hline MID & 37.5-62.5th (motor imagery) & 37.5-62.5th (motor imagery) \\
\hline LOW & $37.5-62.5$ th (motor imagery) & 5-25th (motor imagery) \\
\hline
\end{tabular}

\section{Figure 6 Experimental paradigm}

729 (A) Task instructions and visual SMR-ERD feedback in the contralateral and ipsilateral SM1 730 were provided in the form of computer cursors in a two-dimensional coordinate on a computer screen (upper panel). EEG-triggered TMS timing ranged from 0.5 to $5.5 \mathrm{~s}$ during the MI epoch, and non-triggered TMS (referred to as the failed trial) was delivered in shuffled timing ranging from 5.5 to $6 \mathrm{~s}$ during the MI epoch to determine the influence of spontaneous SMR fluctuations on IHI. Lower panel indicates the experimental overview. The last three HIGH, MID, and LOW sessions were arranged in random order, and in these sessions, participants received visual feedback based on bilateral SMR-ERDs. (B) The predefined target ranges of SMR-ERD were expressed by a blue rectangle on the computer screen in each session. We aimed for participants 
to volitionally increase or decrease (bidirectional) the ipsilateral sensorimotor excitability while maintaining constant contralateral sensorimotor excitability. REST and NoFB sessions were served in order to estimate the individual baseline during rest and MI for the offline analysis.

\section{MEP analysis}

743 For the quality control of MEP analysis, trials were rejected if: (1) coil position was 744 shifted from the optimal orientation and location $\left(>3 \mathrm{~mm}\right.$ and/or $\left.>3^{\circ}\right)$ despite maintaining it during the experiment using the Brainsight TMS navigation system; (2) involuntary muscle contraction in the $250 \mathrm{~ms}$ period before the TMS pulse was observed $(>5 \% \mathrm{MVC}$ ) because of pre-innervation increase in MEP amplitude (Devanne et al., 1997; Hallett, 2007); (3) large trial-by-trial MEP variance (mean \pm 3 SD) were found in order to screen out extreme values (Ruddy et al., 2018). In total, 8.8\% of all trials were excluded from further analysis. Each peak-to-peak MEP amplitude was automatically determined in the remaining trials within $20-45 \mathrm{~ms}$ after the TMS pulse. IHI was defined as the percentage of mean conditioned MEP amplitude over mean unconditioned MEP amplitude $(\mathrm{IHI}=$ conditioned MEP/unconditioned MEP $\times 100 \%)$; therefore, smaller IHI values represent stronger inhibition. EMG and EEG data were processed using the customized analysis scripts on MATLAB R2019a.

\section{Offline EEG analysis}

758 To evaluate the sensorimotor excitability that may influence IHI, pre-processing and time-frequency analyses were performed, and the left and right SMR-ERDs and their laterality were calculated. SMR-ERD in EEG is a reliable surrogate monitoring marker of sensorimotor excitability level for several reasons: (1) SMR-ERD and task-induced increase in blood oxygenation level-dependent signals during MI are co-localized and covaried at SM1 (Yuan et al., 2010); (2) SMR-ERD control is associated with the contribution of SM1 modulated by transcranial direct current stimulation (Soekadar et al., 2015b); and (3) data-driven EEG features discriminating the presence or absence of muscle contraction were predominantly localized in the parieto-temporal regions, indicating SMR-ERD (Hayashi et al., 2019; Iwama et al., 2020). The time segment of interest was from the initiation of the trial to before TMS-triggered time marker of the CS in order to avoid contamination by the TMS artifact (pre-stimulation period). The EEG signal underwent a 1-70-Hz, second-order Butterworth bandpass filter and a 50-Hz notch filter. The EEG signals of all channels were spatially filtered using a common average 
772 reference, which subtracted the average value of the entire electrode montage (the

773 common average) from that of the channel of interest to remove global noise (McFarland

774 et al., 1997; Tsuchimoto et al., 2021). EEG channels in each trial were rejected during

775 further analysis if they contained an amplitude above $100 \mu \mathrm{V}$ (Sanei and Chambers,

776 2013). To examine target-hemisphere-dependent modulation (i.e., difference between the

777 contralateral and ipsilateral sensorimotor activation), LI of the bihemispheric SMR-ERDs

778 was calculated (Seghier, 2008). LI yields a value of 1 or -1 when the activity is purely

779 ipsilateral or contralateral, respectively.

780

\section{Connectivity analysis}

782 To assess interhemispheric functional connectivity at the EEG level, distributed 783 interregional neural communication was calculated. We focused on both the resting epoch 784 (1-5 s) and MI epoch (7 to before stimulation onset) for analysis. To calculate functional connectivity and compensate for long-range synchronization preference, we used the corrected imaginary part of coherence (ciCOH) (Ewald et al., 2012; Hayashi et al., 2020; Vukelić and Gharabaghi, 2015). The details of the following processing for the connectivity analysis can be obtained from our previous work (Hayashi et al., 2020). The ciCOH was obtained by subdividing the resting epoch into 1-s segments with $90 \%$ overlap (31 segments in total) and multiplied with a Hanning window. Then, interhemispheric Network-intensity was computed as follows:

$$
\text { Network intensity }(f)=\sum_{\text {cont }=1}^{7} \sum_{i p s i=1}^{7} \operatorname{ciCOH}_{\text {cont }, i p s i}(f)
$$

793 where Network-intensity is computed between left and right hemisphere, $\sum \sum$ ciCOH is 794 the sum of significant ciCOH values for interhemispheric interaction, cont denotes the 7 795 channels of interest (i.e., C3 and its neighboring 6 channels), ipsi denotes the 7 channels 796 of interest in the opposite hemisphere (i.e., C4 and its neighboring 6 channels), and $f$

797 indicates the frequency of interest (i.e., the predefined alpha frequencies). As a negative 798 control, results of other frequency bands are shown in Supplementary files ("Connectivity results in the other frequency bands").

\section{Correlation analysis}

802 To investigate the association between sensorimotor brain activity at the EEG level and 803 IHI magnitude from multimodal perspectives, correlation analysis was performed. We 
804

805

806

807

808

809

810

811

812

813

814

815

816

\section{Statistical analysis}

818 Statistical analyses were performed using SPSS software (version 27; IBM Corp., 819

820

821

822

823

824

825

826

827

828

829

830

831

832

833

834

835

836

837

used Pearson's correlation since EMG/EEG data showed normal distribution. Withinsubject correlations between bilateral SMR-ERDs and IHI magnitude in each participant and across-subject correlations were performed. Furthermore, in order to examine the neural characteristics depending on the manipulation capability of IHI, we first examined the association between the manipulated effects on IHI calculated from the difference between $\mathrm{HIGH}$ and LOW sessions $\left(\Delta \mathrm{IHI}_{\mathrm{H}-\mathrm{L}}\right)$, and IHI in REST session $\left(\mathrm{IHI}_{\text {rest }}\right)$. Next, the correlations between $\mathrm{IHI}_{\text {rest }}$ and intrinsic EEG profile in NoFB session (i.e., contralateral SMR-ERD, ipsilateral SMR-ERD, and LI) were investigated, respectively. Moreover, we verified whether large-scale resting-state functional connectivity was associated with an effective inhibitory interhemispheric network assessed by IHI. An across-subject Pearson's correlation was applied to identify significant relationships between the $\mathrm{IHI}_{\text {rest }}$ and interhemispheric Network-intensity rest $_{\text {for all participants }}$ Armonk, NY, USA) and MATLAB R2019a. The assumption of normality was verified using the Shapiro-Wilk test. All data were normally distributed $(p>0.05)$ and therefore analyzed with parametric tests. The assumption of sphericity was checked using the Mauchly's test. If the test was significant, a Greenhouse-Geisser correction was applied. For the IHI curves, a one-way rmANOVA for intensities (six levels: 0\% [TS only], 100\%, $110 \%, 120 \%, 130 \%$, and 140\% RMT) and post-hoc two-tailed paired t-tests were performed in MEP amplitude. Modulation effects of bilateral sensorimotor excitabilities due to BCI-base neurofeedback training were evaluated from the 1-s period immediately before stimulation onset. A one-way rmANOVA for sessions (five levels: REST, NoFB, HIGH, MID, and LOW) was performed using the contralateral and ipsilateral SMR-ERDs and LI. Following the one-way rmANOVA, post-hoc two-tailed paired t-tests were performed using the Bonferroni correction for multiple comparisons. For the interhemispheric connectivity during MI, a one-way rmANOVA and post-hoc analysis were applied to Network-intensityм as the same procedure. In addition, to verify the difficulties for neurofeedback sessions, one-way rmANOVA for sessions (HIGH, MID, and LOW) and post-hoc analysis were applied to the number of the triggered trials and mean waiting time for a triggered event from MI onset. For the IHI results, a one-way rmANOVA for sessions (five levels: REST, NoFB, HIGH, MID, and LOW) was performed. Next, for all significant main effects, post-hoc two-tailed paired t-test was 
838 performed using the Bonferroni correction for multiple comparisons for all sessions. We 839 further compared the IHI magnitude across sessions, by normalizing IHI magnitude to 840 baseline (i.e., NoFB session) and calculating the difference between sessions (REST, 841 HIGH, MID, and LOW). The significance level for all statistical tests was set to $p=0.05$. 842 Although analysis of mean IHI magnitude across subjects revealed significant 843 effect of the ipsilateral SMR-ERD (Figure 3B), we further performed mixed-effects 844 analysis (Hussain et al., 2019; Madsen et al., 2019) incorporating the contralateral SMR845 ERD and ipsilateral SMR-ERD as factors in the statistical model to explore the 846 relationships between inhibitory interhemispheric activity and EEG characteristics. The 847 linear mixed-effect model included the contralateral and ipsilateral SMR-ERD as fixed 848 effects, treating the participant factor as a random effect to account for individual 849 variability in IHI magnitude.

850 


\section{Funding}

853 This work was supported by a Grant-in-Aid for Transformative Research Areas (A)

854 (\#20H05923) from the Ministry of Education, Culture, Sports, Science and Technology

855 (MEXT) and Strategic International Brain Science Research Promotion Program

856 (Brain/MINDS Beyond) (\#JP20dm0307022) from the Japan Agency for Medical

857 Research and Development (AMED) to J. Ushiba. In addition, this study was also

858 supported by The Keio University Doctorate Student Grant-in-Aid Program from

859 Ushioda Memorial Fund to M.Hayashi.

860

861 Acknowledgements

862 The authors would like to thank Kohsuke Okada for experimental assistance and to 863 appreciate Sayoko Ishii, Kumi Nanjo, Yoko Mori, Yumiko Kakubari, Shoko Tonomoto, 864 and Aya Kamiya for their technical support during the study. 


\section{References}

Ames KC, Churchland MM. 2019. Motor cortex signals for each arm are mixed across hemispheres and neurons yet partitioned within the population response. eLife 8:e46159. doi:10.7554/eLife.46159

Ang KK, Guan C. 2017. EEG-Based Strategies to Detect Motor Imagery for Control and Rehabilitation. IEEE Transactions on Neural Systems and Rehabilitation Engineering 25:392-401. doi:10.1109/TNSRE.2016.2646763

Ang KK, Guan C, Phua KS, Wang C, Teh I, Chen CW, Chew E. 2012. Transcranial direct current stimulation and EEGbased motor imagery BCI for upper limb stroke rehabilitation. Annu Int Conf IEEE Eng Med Biol Soc 2012:4128-4131. doi:10.1109/EMBC.2012.6346875

Banich MT. 1998. The Missing Link: The Role of Interhemispheric Interaction in Attentional Processing. Brain and Cognition 36:128-157. doi:10.1006/brcg.1997.0950

Boddington LJ, Reynolds JNJ. 2017. Targeting interhemispheric inhibition with neuromodulation to enhance stroke rehabilitation. Brain Stimulation 10:214-222. doi:10.1016/j.brs.2017.01.006

Buch, Weber Cornelia, Cohen Leonardo G., Braun Christoph, Dimyan Michael A., Ard Tyler, Mellinger Jurgen, Caria Andrea, Soekadar Surjo, Fourkas Alissa, Birbaumer Niels. 2008. Think to Move: a Neuromagnetic Brain-Computer Interface (BCl) System for Chronic Stroke. Stroke 39:910-917. doi:10.1161/STROKEAHA.107.505313

Bundy, Souders Lauren, Baranyai Kelly, Leonard Laura, Schalk Gerwin, Coker Robert, Moran Daniel W., Huskey Thy, Leuthardt Eric C. 2017. Contralesional Brain-Computer Interface Control of a Powered Exoskeleton for Motor Recovery in Chronic Stroke Survivors. Stroke 48:1908-1915. doi:10.1161/STROKEAHA.116.016304

Caria A, Weber C, Brötz D, Ramos A, Ticini LF, Gharabaghi A, Braun C, Birbaumer N. 2011. Chronic stroke recovery after combined $\mathrm{BCl}$ training and physiotherapy: a case report. Psychophysiology 48:578-582. doi:10.1111/j.14698986.2010.01117.x

Carter Leno V, Chandler S, White P, Yorke I, Charman T, Pickles A, Simonoff E. 2018a. Alterations in electrophysiological indices of perceptual processing and discrimination are associated with co-occurring emotional and behavioural problems in adolescents with autism spectrum disorder. Mol Autism 9:50. doi:10.1186/s13229-018-0236-2

Carter Leno V, Tomlinson SB, Chang S-AA, Naples AJ, McPartland JC. 2018b. Resting-state alpha power is selectively associated with autistic traits reflecting behavioral rigidity. Sci Rep 8:11982. doi:10.1038/s41598-018-30445-2

Chaudhary U, Birbaumer N, Ramos-Murguialday A. 2016. Brain-computer interfaces for communication and rehabilitation. Nat Rev Neurol 12:513-525. doi:10.1038/nrneurol.2016.113

Chieffo R, Inuggi A, Straffi L, Coppi E, Gonzalez-Rosa J, Spagnolo F, Poggi A, Comi G, Comola M, Leocani L. 2013. Mapping early changes of cortical motor output after subcortical stroke: A transcranial magnetic stimulation study. Brain Stimulation 6:322-329. doi:10.1016/j.brs.2012.06.003

Cohen J. 2013. Statistical Power Analysis for the Behavioral Sciences. Academic Press.

Cohen J. 1992. A power primer. Psychol Bull 112:155-159.

Crone NE, Miglioretti DL, Gordon B, Sieracki JM, Wilson MT, Uematsu S, Lesser RP. 1998. Functional mapping of human sensorimotor cortex with electrocorticographic spectral analysis: I. Alpha and beta event-related desynchronization. Brain 121:2271-2299.

Daskalakis ZJ, Christensen BK, Fitzgerald PB, Roshan L, Chen R. 2002. The mechanisms of interhemispheric inhibition in the human motor cortex. J Physiol 543:317-326. doi:10.1113/jphysiol.2002.017673

Devanne H, Lavoie BA, Capaday C. 1997. Input-output properties and gain changes in the human corticospinal pathway. Exp Brain Res 114:329-338. doi:10.1007/pl00005641

Di Pino G, Pellegrino G, Assenza G, Capone F, Ferreri F, Formica D, Ranieri F, Tombini M, Ziemann U, Rothwell JC, Di Lazzaro V. 2014. Modulation of brain plasticity in stroke: a novel model for neurorehabilitation. Nat Rev Neurol 10:597608. doi:10.1038/nrneurol.2014.162

Dong Y, Dobkin BH, Cen SY, Wu AD, Winstein CJ. 2006. Motor cortex activation during treatment may predict therapeutic gains in paretic hand function after stroke. Stroke 37:1552-1555. doi:10.1161/01.STR.0000221281.69373.4e

Duque J, Hummel F, Celnik P, Murase N, Mazzocchio R, Cohen LG. 2005. Transcallosal inhibition in chronic subcortical stroke. Neuroimage 28:940-946. doi:10.1016/j.neuroimage.2005.06.033 
914 Duque J, Murase N, Celnik P, Hummel F, Harris-Love M, Mazzocchio R, Olivier E, Cohen LG. 2007. Intermanual

915 Differences in movement-related interhemispheric inhibition. J Cogn Neurosci 19:204-213.

916 doi:10.1162/jocn.2007.19.2.204

917 Engelhard B, Ozeri N, Israel Z, Bergman H, Vaadia E. 2013. Inducing Gamma Oscillations and Precise Spike Synchrony

918 by Operant Conditioning via Brain-Machine Interface. Neuron 77:361-375. doi:10.1016/j.neuron.2012.11.015

919 Ewald A, Marzetti L, Zappasodi F, Meinecke FC, Nolte G. 2012. Estimating true brain connectivity from EEG/MEG data invariant to linear and static transformations in sensor space. Neurolmage 60:476-488. doi:10.1016/j.neuroimage.2011.11.084

Faul F, Erdfelder E, Buchner A, Lang A-G. 2009. Statistical power analyses using G*Power 3.1: Tests for correlation and regression analyses. Behavior Research Methods 41:1149-1160. doi:10.3758/BRM.41.4.1149

Ferbert A, Priori A, Rothwell JC, Day BL, Colebatch JG, Marsden CD. 1992. Interhemispheric inhibition of the human motor cortex. J Physiol 453:525-546.

Ferree TC, Luu P, Russell GS, Tucker DM. 2001. Scalp electrode impedance, infection risk, and EEG data quality. Clinical Neurophysiology 112:536-544. doi:10.1016/S1388-2457(00)00533-2

Fetz EE. 2013. Volitional Control of Cortical Oscillations and Synchrony. Neuron 77:216-218. doi:10.1016/j.neuron.2013.01.003

Fries P. 2005. A mechanism for cognitive dynamics: neuronal communication through neuronal coherence. Trends in Cognitive Sciences 9:474-480. doi:10.1016/j.tics.2005.08.011

Ghosh S, Mehta AR, Huang G, Gunraj C, Hoque T, Saha U, Ni Z, Chen R. 2013. Short- and long-latency interhemispheric inhibitions are additive in human motor cortex. J Neurophysiol 109:2955-2962. doi:10.1152/jn.00960.2012

Gilio F, Rizzo V, Siebner HR, Rothwell JC. 2003. Effects on the right motor hand-area excitability produced by lowfrequency rTMS over human contralateral homologous cortex. J Physiol 551:563-573. doi:10.1113/jphysiol.2003.044313

Groppa S, Oliviero A, Eisen A, Quartarone A, Cohen LG, Mall V, Kaelin-Lang A, Mima T, Rossi S, Thickbroom GW, Rossini PM, Ziemann U, Valls-Solé J, Siebner HR. 2012. A practical guide to diagnostic transcranial magnetic stimulation: Report of an IFCN committee. Clinical Neurophysiology 123:858-882. doi:10.1016/j.clinph.2012.01.010

Haegens S, Nácher V, Luna R, Romo R, Jensen O. 2011. a-Oscillations in the monkey sensorimotor network influence discrimination performance by rhythmical inhibition of neuronal spiking. PNAS 108:19377-19382. doi:10.1073/pnas. 1117190108

Hallett M. 2007. Transcranial magnetic stimulation: a primer. Neuron 55:187-199. doi:10.1016/j.neuron.2007.06.026

Harris-Love ML, Perez MA, Chen R, Cohen LG. 2007. Interhemispheric Inhibition in Distal and Proximal Arm Representations in the Primary Motor Cortex. Journal of Neurophysiology 97:2511-2515. doi:10.1152/jn.01331.2006

Hayashi M, Mizuguchi N, Tsuchimoto S, Ushiba J. 2021. Neurofeedback of Scalp Bi-Hemispheric EEG Sensorimotor Rhythm Guides Hemispheric Activation of Sensorimotor Cortex in the Targeted Hemisphere In: Guger C, Allison BZ, Tangermann M, editors. Brain-Computer Interface Research: A State-of-the-Art Summary 9, SpringerBriefs in Electrical and Computer Engineering. Cham: Springer International Publishing. pp. 25-38. doi:10.1007/978-3-030-60460-8_3

Hayashi M, Mizuguchi N, Tsuchimoto S, Ushiba J. 2020. Neurofeedback of scalp bi-hemispheric EEG sensorimotor rhythm guides hemispheric activation of sensorimotor cortex in the targeted hemisphere. Neurolmage 223:117298. doi:10.1016/j.neuroimage.2020.117298

Hayashi M, Tsuchimoto S, Mizuguchi N, Miyatake M, Kasuga S, Ushiba J. 2019. Two-stage regression of high-density scalp electroencephalograms visualizes force regulation signaling during muscle contraction. J Neural Eng 16:056020. doi:10.1088/1741-2552/ab221a

He S, Everest-Phillips C, Clouter A, Brown P, Tan H. 2020. Neurofeedback-Linked Suppression of Cortical $\beta$ Bursts Speeds Up Movement Initiation in Healthy Motor Control: A Double-Blind Sham-Controlled Study. J Neurosci 40:40214032. doi:10.1523/JNEUROSCI.0208-20.2020

Hofer S, Frahm J. 2006. Topography of the human corpus callosum revisited: Comprehensive fiber tractography using diffusion tensor magnetic resonance imaging. Neurolmage 32:989-994. doi:10.1016/j.neuroimage.2006.05.044 
961

Hummel FC, Cohen LG. 2006. Non-invasive brain stimulation: a new strategy to improve neurorehabilitation after stroke? The Lancet Neurology 5:708-712. doi:10.1016/S1474-4422(06)70525-7

Hussain SJ, Claudino L, Bönstrup M, Norato G, Cruciani G, Thompson R, Zrenner C, Ziemann U, Buch E, Cohen LG. 2019. Sensorimotor Oscillatory Phase-Power Interaction Gates Resting Human Corticospinal Output. Cerebral Cortex 29:3766-3777. doi:10.1093/cercor/bhy255

Iwama S, Tsuchimoto S, Hayashi M, Mizuguchi N, Ushiba J. 2020. Scalp electroencephalograms over ipsilateral sensorimotor cortex reflect contraction patterns of unilateral finger muscles. Neuroimage 222:117249. doi:10.1016/j.neuroimage.2020.117249

Kober SE, Witte M, Grinschgl S, Neuper C, Wood G. 2018. Placebo hampers ability to self-regulate brain activity: A double-blind sham-controlled neurofeedback study. Neuroimage 181:797-806. doi:10.1016/j.neuroimage.2018.07.025

Liew S-L, Rana M, Cornelsen S, Fortunato de Barros Filho M, Birbaumer N, Sitaram R, Cohen LG, Soekadar SR. 2016. Improving Motor Corticothalamic Communication After Stroke Using Real-Time fMRI Connectivity-Based Neurofeedback. Neurorehabil Neural Repair 30:671-675. doi:10.1177/1545968315619699

Madsen KH, Karabanov AN, Krohne LG, Safeldt MG, Tomasevic L, Siebner HR. 2019. No trace of phase: Corticomotor excitability is not tuned by phase of pericentral mu-rhythm. Brain Stimulation 12:1261-1270. doi:10.1016/j.brs.2019.05.005

McFarland DJ, McCane LM, David SV, Wolpaw JR. 1997. Spatial filter selection for EEG-based communication. Electroencephalogr Clin Neurophysiol 103:386-394.

Meyer B-U, Röricht S, von Einsiedel HG, Kruggel F, Weindl A. 1995. Inhibitory and excitatory interhemispheric transfers between motor cortical areas in normal humans and patients with abnormalities of the corpus callosum. Brain 118:429440. doi:10.1093/brain/118.2.429

Moher D, Schulz KF, Altman D, Group for the C. 2001. The CONSORT Statement: Revised recommendations for omproving the quality of reports of parallel-group randomized trials. JAMA 285:1987-1991. doi:10.1001/jama.285.15.1987

Murase N, Duque J, Mazzocchio R, Cohen LG. 2004. Influence of interhemispheric interactions on motor function in chronic stroke. Ann Neurol 55:400-409. doi:10.1002/ana.10848

Neuper C, Pfurtscheller G. 2001. Event-related dynamics of cortical rhythms: frequency-specific features and functional correlates. Int J Psychophysiol 43:41-58.

Neuper C, Scherer R, Reiner M, Pfurtscheller G. 2005. Imagery of motor actions: Differential effects of kinesthetic and visual-motor mode of imagery in single-trial EEG. Cognitive Brain Research 25:668-677. doi:10.1016/j.cogbrainres.2005.08.014

Neuper C, Wörtz M, Pfurtscheller G. 2006. ERD/ERS patterns reflecting sensorimotor activation and deactivation. Prog Brain Res 159:211-222. doi:10.1016/S0079-6123(06)59014-4

Ni Z, Gunraj C, Nelson AJ, Yeh I-J, Castillo G, Hoque T, Chen R. 2009. Two Phases of Interhemispheric Inhibition between Motor Related Cortical Areas and the Primary Motor Cortex in Human. Cerebral Cortex 19:1654-1665. doi:10.1093/cercor/bhn201

Ni Z, Müller-Dahlhaus F, Chen R, Ziemann U. 2011. Triple-pulse TMS to study interactions between neural circuits in human cortex. Brain Stimulation 4:281-293. doi:10.1016/j.brs.2011.01.002

Notturno F, Marzetti L, Pizzella V, Uncini A, Zappasodi F. 2014. Local and remote effects of transcranial direct current stimulation on the electrical activity of the motor cortical network. Human Brain Mapping 35:2220-2232. doi:10.1002/hbm.22322

Oldfield RC. 1971. The assessment and analysis of handedness: The Edinburgh inventory. Neuropsychologia 9:97-113. doi:10.1016/0028-3932(71)90067-4

Palmer LM, Schulz JM, Murphy SC, Ledergerber D, Murayama M, Larkum ME. 2012. The cellular basis of GABA(B)mediated interhemispheric inhibition. Science 335:989-993. doi:10.1126/science.1217276

Peña-Gómez C, Sala-Lonch R, Junqué C, Clemente IC, Vidal D, Bargalló N, Falcón C, Valls-Solé J, Pascual-Leone Á, Bartrés-Faz D. 2012. Modulation of large-scale brain networks by transcranial direct current stimulation evidenced by 
bioRxiv preprint doi: https://doi.org/10.1101/2021.12.15.472763; this version posted December 29, 2021. The copyright holder for this preprint (which was not certified by peer review) is the author/funder. All rights reserved. No reuse allowed without permission.

resting-state functional MRI. Brain Stimul 5:252-263. doi:10.1016/j.brs.2011.08.006

Pereira J, Direito B, Sayal A, Ferreira C, Castelo-Branco M. 2019. Self-Modulation of Premotor Cortex Interhemispheric Connectivity in a Real-Time Functional Magnetic Resonance Imaging Neurofeedback Study Using an Adaptive Approach. Brain Connectivity 9:662-672. doi:10.1089/brain.2019.0697

Pfurtscheller G. 2001. Functional brain imaging based on ERD/ERS. Vision Research 41:1257-1260. doi:10.1016/S00426989(00)00235-2

Pfurtscheller G, Brunner C, Schlögl A, Lopes da Silva FH. 2006. Mu rhythm (de)synchronization and EEG single-trial classification of different motor imagery tasks. Neuroimage 31:153-159. doi:10.1016/j.neuroimage.2005.12.003

Pfurtscheller G, Neuper C. 1997. Motor imagery activates primary sensorimotor area in humans. Neurosci Lett 239:6568.

Picazio S, Veniero D, Ponzo V, Caltagirone C, Gross J, Thut G, Koch G. 2014. Prefrontal control over motor cortex cycles at beta frequency during movement inhibition. Curr Biol 24:2940-2945. doi:10.1016/j.cub.2014.10.043

Ramos-Murguialday A, Broetz D, Rea M, Läer L, Yilmaz Ö, Brasil FL, Liberati G, Curado MR, Garcia-Cossio E, Vyziotis A, Cho W, Agostini M, Soares E, Soekadar S, Caria A, Cohen LG, Birbaumer N. 2013. Brain-Machine-Interface in Chronic Stroke Rehabilitation: A Controlled Study. Ann Neurol 74:100-108. doi:10.1002/ana.23879

Robertson MM, Furlong S, Voytek B, Donoghue T, Boettiger CA, Sheridan MA. 2019. EEG power spectral slope differs by ADHD status and stimulant medication exposure in early childhood. Journal of Neurophysiology 122:2427-2437. doi:10.1152/jn.00388.2019

Ros T, Enriquez-Geppert S, Zotev V, Young KD, Wood G, Whitfield-Gabrieli S, Wan F, Vuilleumier P, Vialatte F, Van De Ville D, Todder D, Surmeli T, Sulzer JS, Strehl U, Sterman MB, Steiner NJ, Sorger B, Soekadar SR, Sitaram R, Sherlin LH, Schönenberg M, Scharnowski F, Schabus M, Rubia K, Rosa A, Reiner M, Pineda JA, Paret C, Ossadtchi A, Nicholson AA, Nan W, Minguez J, Micoulaud-Franchi J-A, Mehler DMA, Lührs M, Lubar J, Lotte F, Linden DEJ, LewisPeacock JA, Lebedev MA, Lanius RA, Kübler A, Kranczioch C, Koush Y, Konicar L, Kohl SH, Kober SE, Klados MA, Jeunet C, Janssen TWP, Huster RJ, Hoedlmoser K, Hirshberg LM, Heunis S, Hendler T, Hampson M, Guggisberg AG, Guggenberger R, Gruzelier JH, Göbel RW, Gninenko N, Gharabaghi A, Frewen P, Fovet T, Fernández T, Escolano C, Ehlis A-C, Drechsler R, Christopher deCharms R, Debener S, De Ridder D, Davelaar EJ, Congedo M, Cavazza M, Breteler MHM, Brandeis D, Bodurka J, Birbaumer N, Bazanova OM, Barth B, Bamidis PD, Auer T, Arns M, Thibault RT. 2020. Consensus on the reporting and experimental design of clinical and cognitive-behavioural neurofeedback studies (CRED-nf checklist). Brain. doi:10.1093/brain/awaa009

Rossi S, Hallett M, Rossini PM, Pascual-Leone A. 2009. Safety, ethical considerations, and application guidelines for the use of transcranial magnetic stimulation in clinical practice and research. Clinical Neurophysiology 120:2008-2039. doi:10.1016/j.clinph.2009.08.016

Rossini PM, Barker AT, Berardelli A, Caramia MD, Caruso G, Cracco RQ, Dimitrijević MR, Hallett M, Katayama Y, Lücking $\mathrm{CH}$, Maertens de Noordhout AL, Marsden CD, Murray NMF, Rothwell JC, Swash M, Tomberg C. 1994. Non-invasive electrical and magnetic stimulation of the brain, spinal cord and roots: basic principles and procedures for routine clinical application. Report of an IFCN committee. Electroencephalography and Clinical Neurophysiology 91:79-92. doi:10.1016/0013-4694(94)90029-9

Rossini PM, Burke D, Chen R, Cohen LG, Daskalakis Z, Di lorio R, Di Lazzaro V, Ferreri F, Fitzgerald PB, George MS, Hallett M, Lefaucheur JP, Langguth B, Matsumoto H, Miniussi C, Nitsche MA, Pascual-Leone A, Paulus W, Rossi S, Rothwell JC, Siebner HR, Ugawa Y, Walsh V, Ziemann U. 2015. Non-invasive electrical and magnetic stimulation of the brain, spinal cord, roots and peripheral nerves: Basic principles and procedures for routine clinical and research application. An updated report from an I.F.C.N. Committee. Clinical Neurophysiology 126:1071-1107. doi:10.1016/j.clinph.2015.02.001

Ruddy K, Balsters J, Mantini D, Liu Q, Kassraian-Fard P, Enz N, Mihelj E, Chander BS, Soekadar SR, Wenderoth N. 2018. Neural activity related to volitional regulation of cortical excitability. eLife. doi:10.7554/eLife.40843

Safeldt MG, Tomasevic L, Karabanov A, Siebner H, Madsen KH. 2017. Towards brain-state dependent transcranial magnetic stimulation: Targeting the phase of oscillatory neocortical activity with singe-pulse TMS. Brain Stimulation: Basic, Translational, and Clinical Research in Neuromodulation 10:449-450. doi:10.1016/j.brs.2017.01.323 
Sanei S, Chambers JA. 2013. Introduction to EEGEEG Signal Processing. John Wiley \& Sons, Ltd. pp. 1-34. doi:10.1002/9780470511923.ch1

Sauseng P, Klimesch W, Gerloff C, Hummel FC. 2009. Spontaneous locally restricted EEG alpha activity determines cortical excitability in the motor cortex. Neuropsychologia 47:284-288. doi:10.1016/j.neuropsychologia.2008.07.021

1060

Schulz H, Ubelacker T, Keil J, Müller N, Weisz N. 2014. Now I am ready-now i am not: The influence of pre-TMS oscillations and corticomuscular coherence on motor-evoked potentials. Cereb Cortex 24:1708-1719. doi:10.1093/cercor/bht024

Seghier ML. 2008. Laterality index in functional MRI: Methodological issues. Magn Reson Imaging 26:594-601. doi:10.1016/j.mri.2007.10.010

Sitaram R, Ros T, Stoeckel L, Haller S, Scharnowski F, Lewis-Peacock J, Weiskopf N, Blefari ML, Rana M, Oblak E, Birbaumer N, Sulzer J. 2017. Closed-loop brain training: the science of neurofeedback. Nature Reviews Neuroscience 18:86-100. doi:10.1038/nrn.2016.164

Soekadar SR, Birbaumer N, Slutzky MW, Cohen LG. 2015a. Brain-machine interfaces in neurorehabilitation of stroke. Neurobiology of Disease 83:8. doi:10.1016/j.nbd.2014.11.025

Soekadar SR, Witkowski M, Birbaumer N, Cohen LG. 2015b. Enhancing Hebbian Learning to Control Brain Oscillatory Activity. Cereb Cortex 25:2409-2415. doi:10.1093/cercor/bhu043

Stefanou M-I, Desideri D, Belardinelli P, Zrenner C, Ziemann U. 2018. Phase synchronicity of $\mu$-rhythm determines efficacy of interhemispheric communication between human motor cortices. $J$ Neurosci 1470-18. doi:10.1523/JNEUROSCI.1470-18.2018

Takemi M, Maeda T, Masakado Y, Siebner HR, Ushiba J. 2018. Muscle-selective disinhibition of corticomotor representations using a motor imagery-based brain-computer interface. Neurolmage 183:597-605. doi:10.1016/j.neuroimage.2018.08.070

Takemi M, Masakado Y, Liu M, Ushiba J. 2015. Sensorimotor event-related desynchronization represents the excitability of human spinal motoneurons. Neuroscience 297:58-67. doi:10.1016/j.neuroscience.2015.03.045

1080

Takemi M, Masakado Y, Liu M, Ushiba J. 2013. Event-related desynchronization reflects downregulation of intracortical inhibition in human primary motor cortex. J Neurophysiol 110:1158-1166. doi:10.1152/jn.01092.2012

Thies M, Zrenner C, Ziemann U, Bergmann TO. 2018. Sensorimotor mu-alpha power is positively related to corticospinal excitability. Brain Stimulation 11:1119-1122. doi:10.1016/j.brs.2018.06.006

1084

Thompson AK, Chen XY, Wolpaw JR. 2009. Acquisition of a Simple Motor Skill: Task-Dependent Adaptation Plus LongTerm Change in the Human Soleus H-Reflex. J Neurosci 29:5784-5792. doi:10.1523/JNEUROSCI.4326-08.2009

Tsuchimoto S, Shibusawa S, Iwama S, Hayashi M, Okuyama K, Mizuguchi N, Kato K, Ushiba J. 2021. Use of common average reference and large-Laplacian spatial-filters enhances EEG signal-to-noise ratios in intrinsic sensorimotor activity. J Neurosci Methods 353:109089. doi:10.1016/j.jneumeth.2021.109089

Tsutsumi R, Shirota Y, Ohminami S, Terao Y, Ugawa Y, Hanajima R. 2012. Conditioning intensity-dependent interaction between short-latency interhemispheric inhibition and short-latency afferent inhibition. J Neurophysiol 108:1130-1137. doi:10.1152/jn.00300.2012

Varela F, Lachaux J-P, Rodriguez E, Martinerie J. 2001. The brainweb: Phase synchronization and large-scale integration. Nature Reviews Neuroscience 2:229-239. doi:10.1038/35067550

Vukelić M, Gharabaghi A. 2015. Oscillatory entrainment of the motor cortical network during motor imagery is modulated by the feedback modality. Neurolmage 111:1-11. doi:10.1016/j.neuroimage.2015.01.058

Weiskopf N, Mathiak K, Bock SW, Scharnowski F, Veit R, Grodd W, Goebel R, Birbaumer N. 2004. Principles of a braincomputer interface $(\mathrm{BCl})$ based on real-time functional magnetic resonance imaging (fMRI). IEEE Trans Biomed Eng 51:966-970. doi:10.1109/TBME.2004.827063

Williams JA, Pascual-Leone A, Fregni F. 2010. Interhemispheric modulation induced by cortical stimulation and motor training. Phys Ther 90:398-410. doi:10.2522/ptj.20090075

1101 Xu J, Branscheidt M, Schambra H, Steiner L, Widmer M, Diedrichsen J, Goldsmith J, Lindquist M, Kitago T, Luft AR, 1102 Krakauer JW, Celnik PA, SMARTS Study Group. 2019. Rethinking interhemispheric imbalance as a target for stroke 

neurorehabilitation. Ann Neurol 85:502-513. doi:10.1002/ana.25452

Yuan H, Liu T, Szarkowski R, Rios C, Ashe J, He B. 2010. Negative covariation between task-related responses in alpha/beta-band activity and BOLD in human sensorimotor cortex: an EEG and fMRI study of motor imagery and

1106 movements. Neuroimage 49:2596-2606. doi:10.1016/j.neuroimage.2009.10.028

Zarkowski P, Shin CJ, Dang T, Russo J, Avery D. 2006. EEG and the Variance of Motor Evoked Potential Amplitude. Clin EEG Neurosci 37:247-251. doi:10.1177/155005940603700316

1109

Zrenner C, Desideri D, Belardinelli P, Ziemann U. 2018. Real-time EEG-defined excitability states determine efficacy of

1110 TMS-induced plasticity in human motor cortex. Brain Stimulation 11:374-389. doi:10.1016/j.brs.2017.11.016 